\title{
Ordered structures in ternary hep alloys
}

\author{
ASHOK KUMAR SINGH and SHRIKANT LELE \\ Department of Metallurgical Engineering, Banaras Hindu University, Varanasi 221005 , \\ India
}

MS received 12 July 1990; revised 15 January 1991

\begin{abstract}
A study of ordered structures in ternary hep alloys has been undertaken. For this, the hep structure has been divided into several sublattices and used to generate ordered structures with three types of atoms. Nine ground-state ordered structures have been identified on the basis of maximum or minimum number of $A B, B C$ and $C A$ bonds. Complete crystallographic details about these structures have been worked out. In an alternate approach, ordered ternary structures were obtained by populating 8 types of sublattices (which generate $\mathrm{Ti}_{3} \mathrm{Al}$ structure) with three types of atoms. Thus, fortyeight ordered structures were found. For each structure, complete structural details have also been worked out, some of which are reported. Configurational energy of each structure has been calculated using pairwise interactions up to third neighbour distances. To gain insight regarding low-energy structures, some assumptions were utilized to reduce the number of independent parameters in the energy expressions and their consequences explored. Two types of degenerate situations have been observed. One type of degeneracy occurs for ideal hcp alloys where only first neighbour interactions are considered. Another degenerate situation occurs for non-ideal hcp alloys where interactions are considered up to third neighbour distances.
\end{abstract}

Keywords. Configurational energy; sublattices; ternary hep ordered ground-state structures; low-energy ternary hcp-ordered structures.

\section{Introduction}

The recent spurt of interest in ternary intermetallics based on $\mathrm{Ti}_{3} \mathrm{Al}$ has led to a recognition of the need to understand the thermodynamics of mixing of ternary hcp alloys. Differences in the thermodynamic stability of such phases are expected to lead to differences in their phase transformation behaviour and thus to different paths of microstructural evolution. Needless to add, these differences would significantly affect mechanical properties. Thus ternary additions and their consequent effects would play a key role in the selection of such intermetallics. We have earlier obtained the ordered ground-state structures in binary hcp (hexagonal close-packed) alloys by division of the hcp atomic sites into several sublattices (Singh and Lele 1990). Different structures are obtained by populating the atomic sites by two types of atoms in appropriate proportions. Energies of these structures (having non-ideal axial ratio) are obtained by considering pairwise interactions up to third neighbour distances. Although this method is not an exhaustive procedure for generating superstructures, it is a powerful method for an initial survey of the various possibilities.

Richards and Cahn (1971) proposed a somewhat similar method for constructing superlattice cells using three non-coplanar basis vectors which join atoms at second/third neighbour distances and applied it to fcc and bec structures. Since, in hep alloys both the second and third neighbour vectors are not lattice vectors for the fundamental structure, this method is not applicable to such alloys. Several other 
methods (Allen and Cahn 1972; Kanamori 1966; Kudō and Katsura 1976) have been developed to establish ground state structures for binary bec and fcc alloys.

The present work is concerned with the preliminary extension of the method of sublattices to ternary hcp alloys. Ordered ternary ground-state structures have been proposed on the basis of the occurrence of maximum or minimum number of $A B$, $B C$ and $C A$ bonds at first, second and third neighbour distances. An attempt is also made to identify the low-energy structures under some simplifying assumptions with the 8 sublattice division which generates the $\mathrm{Ti}_{3} \mathrm{Al}\left(\mathrm{DO}_{19}\right)$ structure (Singh and Lele 1990). This information is expected to be useful in ultimately establishing the ground-state structures for ternary hcp alloys.

\section{Model}

The present work is concerned with ternary hcp alloys having constituents $A, B$ and $C$. We have used the Ising model and considered interactions up to third neighbour distances with the following assumptions:

(i) The energy is solely a function of pairwise interactions between the $A, B$ and $C$ atoms at distances up to third neighbours.

(ii) Vibrational and size effects are not taken into account.

(iii) Surface and interfacial effects are also not considered.

(iv) Vacant lattice sites are not allowed. Each lattice site is occupied by an atom of any one of the constituents $A, B$ or $C$.

The hcp structure with non-ideal axial ratio is divided into several sublattices ranging from a minimum of 6 to a maximum of 28. The details of the method of sublattice division have been described in our earlier studies (Singh and Lele 1990). Briefly the procedure is as follows. (i) Every point of a certain type of sublattice has as first, second and third nearest neighbours only points belonging to other types of sublattices. (ii) The division is such that all the sublattices are identical. As shown earlier (Singh and Lele 1990), sublattice divisions ranging from 6 to 14 are sufficient to generate most of the ground-state structures for binary alloys. We consider up to 14 sublattice divisions for the determination of ground-state structures on the basis of the existence of maximum or minimum number of $A B, B C$ and $C A$ bonds. The [001] projections illustrating the sublattice divisions are shown in figures 1 to 5 .

\section{Configurational energy}

Let us consider a ternary hep alloy where the structure is divided into $M$ sublattices ( $M=6$ to 14). Let the alloy consist of lattice sites occupied by $N$ atoms with $N_{A}, N_{B}$ and $N_{c}$ atoms of $A, B$ and $C$ types respectively.

$$
N=\sum_{\alpha} N_{\alpha}, \alpha=A, B \text { and } C \text {. }
$$

Further, let $P_{\alpha}^{i}(i=1$ to $M)$ denote the probability of finding an $\alpha(A, B$ or $C)$ type atom at any site of sublattice $i$. This is related to the number, $N_{\alpha}^{i}$, of $\alpha$ atoms on sublattice $i$ through 


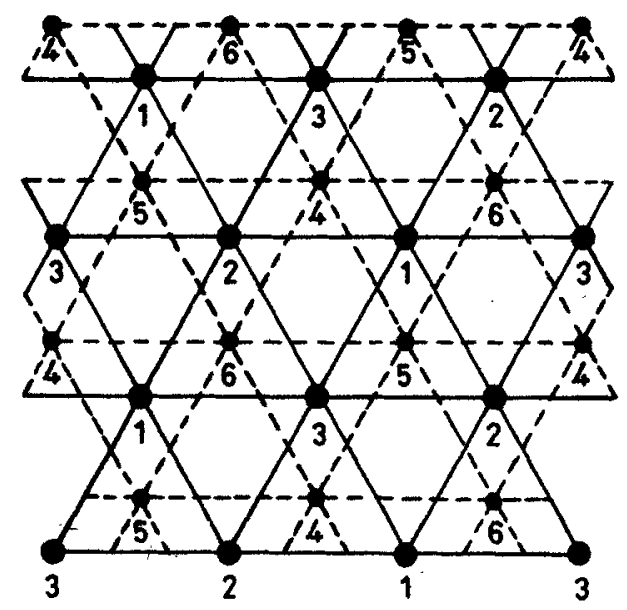

Figure 1. Basal plane projection of hcp structure showing subdivision of the atomic sites into six sublattices numbered 1 to 6 . Large and small circles correspond to sites in alternate layers along the $c$-axis.

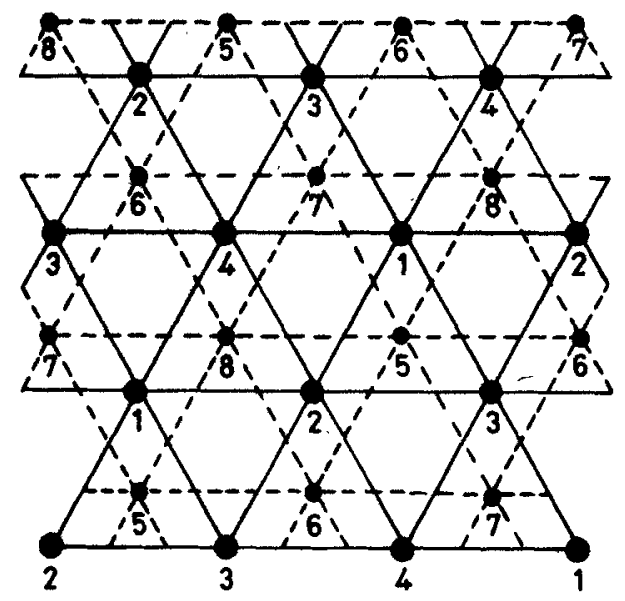

(a) type 1

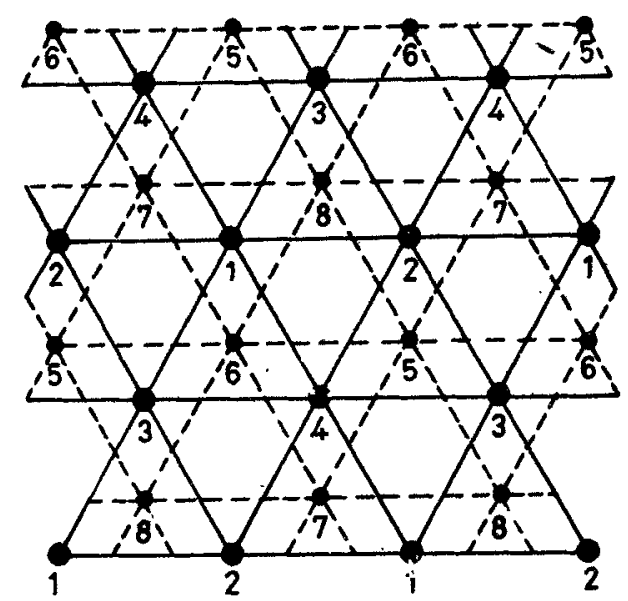

(b) type 2

Figure 2. Basal plane projection of hep structure showing subdivision of the atomic sites into eight sublattices numbered 1 to 8 .

$$
P_{\alpha}^{i}=N_{\alpha}^{i} /(N / M) \text {. }
$$

It follows that

$$
\Sigma P_{\alpha}^{i}=1 \text {. }
$$

The atomic concentration $C_{\alpha}$ can be defined as

$$
C_{\alpha}=(1 / M) \sum_{i=1}^{M} N_{\alpha}^{i} /(N / M) \text {. }
$$

Let us first consider the case of eight sublattice divisions which generate $\mathrm{Ti}_{3} \mathrm{Al}$ 


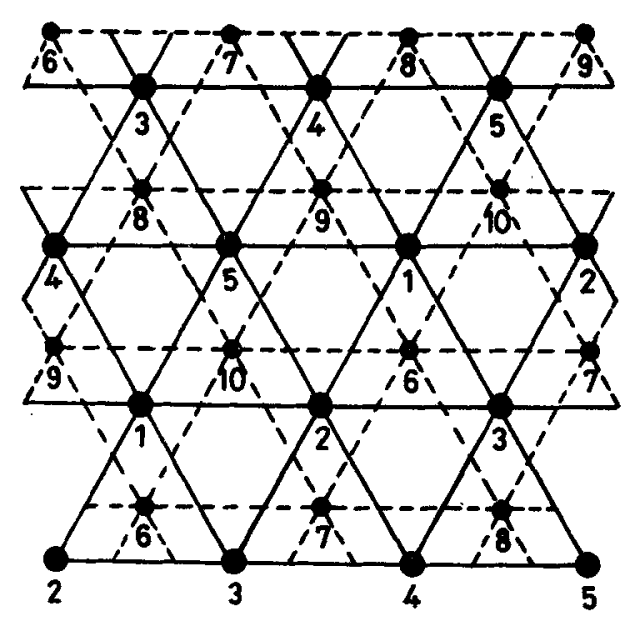

Figure 3. Basal plane projection of hcp structure showing subdivision of the atomic sites into 10 sublattices numbered 1 to 10 .

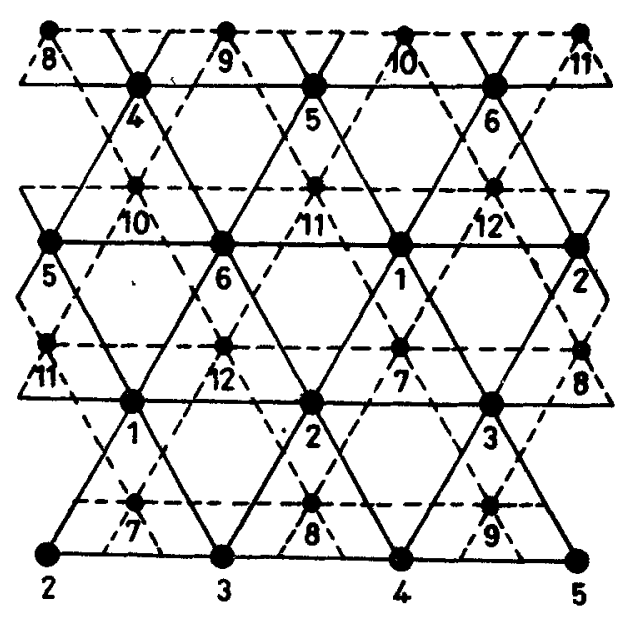

(a) type 1

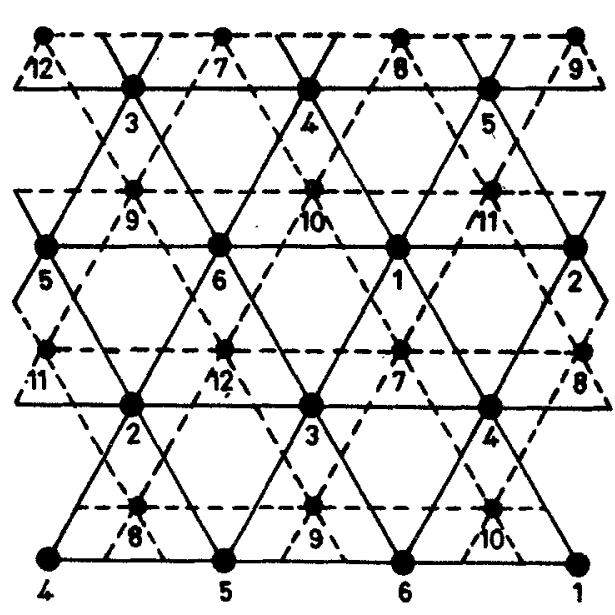

(b) type 2

Figure 4. Basal plane projection of hep structure showing subdivision of the atomic sites into 12 sublattices numbered 1 to 12 .

structure (figure $2 \mathrm{~b}$ ). It is clear from this figure that an atom situated on any one sublattice is surrounded by six sites at first, second and third neighbour distances. For example, an atơm on sublattice 1 is surrounded by 2 first neighbour atoms each on sublattices 2,3 and 4, by 2 second neighbour atoms each on sublattices 6,7 and 8 (for axial ratios greater than the ideal value) and 6 third neighbour atoms on sublattice 5 . For axial ratios less than the ideal one, the roles of first and second neighbour sites are reversed.

Let the number of $k$ th $(k=1$ to 3$)$ neighbour $A-B, B-C$ and $C-A$ bonds be $q_{k}$, $r_{k}$ and $s_{k}$ respectively. The number of $k$ th neighbour $A-B$ bonds, $q_{k}$, can be determined as follows. The number of $A$ atoms on the sublattice 1 is $(N / 8) P_{A}^{1}$. Any $A$ atom is surrounded by $B$ atoms at first neighbour distances on sublattices 2,3 


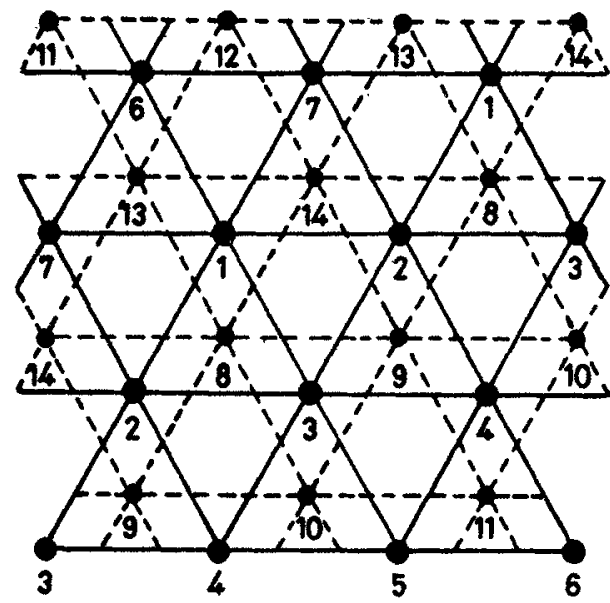

(a) type 1

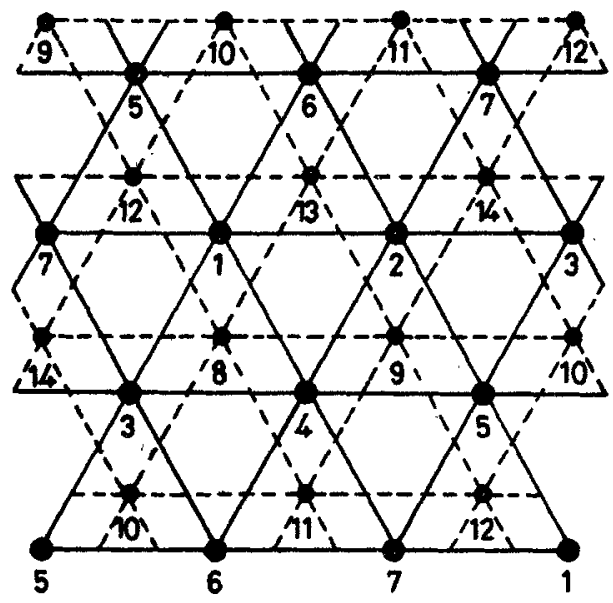

(b) type 2

Figure 5. Basal plane projection of hep structure showing subdivision of the atomic sites into 14 sublattices numbered 1 to 14 .

and 4 with probability $\left(P_{B}^{2}+P_{B}^{3}+P_{B}^{4}\right)$. Thus, the number of $A-B$ bonds at first neighbour distance for this case is $(N / 8) P_{A}^{1}\left(P_{B}^{2}+P_{B}^{3}+P_{B}^{4}\right)$. Considering all similar cases and summing, we have:

$$
q_{1}=(N / 4)\left[\sum_{i=1}^{4} \sum_{\substack{j=1 \\ i \neq j}}^{4} P_{A}^{i} P_{B}^{j}+\sum_{i=5}^{8} \sum_{\substack{j=5 \\ i \neq j}}^{8} P_{A}^{i} P_{B}^{j}\right] .
$$

In a similar way the second and third neighbour $A-B$ bonds can be shown to be:

$$
\begin{aligned}
& q_{2}=(N / 4)\left[\sum_{i=1}^{4} \sum_{\substack{j=5 \\
j \neq i+4}}^{8} P_{A}^{i} P_{B}^{j}+\sum_{i=5}^{8} \sum_{\substack{j=1 \\
j \neq i-4}}^{4} P_{A}^{i} P_{B}^{j}\right] \\
& q_{3}=(N / 4)\left[\sum_{i=1}^{4} P_{A}^{i} P_{B}^{i+4}+\sum_{i=5}^{8} P_{A}^{i} P_{B}^{i-4}\right] .
\end{aligned}
$$

An analogous exercise can be undertaken to determine the number of $B-C\left(r_{1}, r_{2}\right.$ and $\left.r_{3}\right)$ and $C-A\left(s_{1}, s_{2}\right.$ and $\left.s_{3}\right)$ bonds. Expressions for $B-C$ and $C-A$ bonds respectively can be obtained by cyclic and anticyclic permutation of $A, B, C$ in equations (5) to (7). The configurational energy of mixing by definition is:

$$
E=(1 / 2) \sum_{k=1}^{3}\left(q_{k} W_{A B}^{k}+r_{k} W_{B C}^{k}+s_{k} W_{C A}^{k}\right)
$$

where the interchange energies are given by:

$$
\begin{aligned}
W_{A B}^{k} & =2 V_{A B}^{k}-V_{A A}^{k}-V_{B B}^{k}, \\
\text { and } \quad W_{B C}^{k} & =2 V_{B C}^{k}-V_{B B}^{k}-V_{C C}^{k}, \\
W_{C A}^{k} & =2 V_{C A}^{k}-V_{C C}^{k}-V_{A A}^{k},
\end{aligned}
$$


and $V_{A B}^{k}, V_{B C}^{k}, V_{C A}^{k}, V_{A A}^{k}, B_{B B}^{k}$ and $V_{C C}^{k}$ are the bond energies for the $k$ th $(k=1$ to 3$)$ neighbour $A-B, B-C, C-A, A-A, B-B$ and $C-C$ bonds respectively.

Substituting the values of $q_{1}, q_{2}, q_{3}, r_{1}, r_{2}, r_{3}, s_{1}, s_{2}$ and $s_{3}$ in (8) one can obtain the configurational energy of mixing for division into eight sublattices. A similar procedure has been adopted to arrive at the configurational energy for other sublattice divisions.

\section{Ground state structures and their energies}

To identify structures with minimum energy for different interchange energy ratios is a formidable task in this case owing to the large number of independent interchange energies. In the case of binary alloys, there are only three such variables. In contrast, for ternary alloys, there are nine variables. Minimization of energy can only be performed in a 9-dimensional hyperspace. The application of the minimum energy criterion for different interchange energies will yield the number of ordered ground state structures.

It is obvious from (8) that if the values of $q_{k}, r_{k}$ and $s_{k}$ are either maximum or minimum, then the corresponding energy of the ordered structures is minimum for a suitable range of values of the interchange energies. The values of $q_{k}, r_{k}$ and $s_{k}$ for $k$ th neighbour distances must lie in the range

$$
\begin{aligned}
& 0 \leqslant q_{k} / N \leqslant Z_{k} C_{B}, \\
& 0 \leqslant r_{k} / N \leqslant Z_{k} C_{C}, \\
& 0 \leqslant s_{k} / N \leqslant Z_{k} C_{C},
\end{aligned}
$$

where $C_{B}$ and $C_{C}$ are the concentrations of $B$ and $C$ types of atoms respectively and $Z_{k}$ the co-ordination number of $k$ th shell. The above discussion is based on the assumption that $C_{A} \geqslant C_{B} \geqslant C_{C}$. For the lower limit of $A B, B C$ and $C A$ bonds, the minority atoms are completely surrounded by like atoms at the $k$ th neighbour distances and for the upper limit by unlike atoms. To attain the minimum energy for maximum values of $q_{k}, r_{k}$ and $s_{k}(k=1$ to 3$)$, the corresponding interchange energies $W_{A B}^{k}, W_{B C}^{k}$ and $W_{C A}^{k}$ must be negative. On the other hand, for minimum $q_{k}$, $r_{k}$ and $s_{k}$, the interchange energies are positive.

The procedure is briefly described for $A_{12} B C$ structure in what follows. An atom on sublattice 5 (occupied by $C$ atoms) (figure 5 a) is surrounded by six first neighbour atoms on sublattices 3,4,6 and 7 (one 3, two 4, two 6 and one 7) respectively. Similarly, at second and third neighbour distances, it is surrounded by six atoms on sublattices 10,11 and 12 (two on each) and 9,11 and 13 (two on each) respectively. For $A_{12} B C$ stoichiometric structure, we can place $B$ atoms either on sublattice 1 or 2 in order to have the minimum number of $B C$ bonds as also the maximum number of $A B$ and $C A$ bonds at first neighbour distances. Alternately, the $B$ atoms can be placed either on sublattice 8 or 14 to have minimum number of $B C$ bonds as well as maximum number of $A B$ and $C A$ bonds at second or third neighbour distances. This exhausts all possibilities for $A_{12} B C$ structure and results in four crystallographically different structures with the same energy. The second type of subdivision into 14 sublattices (figure 5b) yields only one such possibility for the same stoichiometry. A similar method has been adopted for all possible stoichiometries using appropriate sublattice division. On the basis of the above 
discussion, we have found nine energetically distinct structures with stoichiometries $A_{12} B C, A_{7} B_{6} C, A_{10} B C, A_{6} B_{5} C, A_{5} B_{4} C, A_{6} B C, A_{4} B_{3} C, A_{7} B_{5} C_{2}$ and $A_{3} B_{2} C$ to have minimum energies and thus to represent ground state. The energies of these structures are given in table 1 while the nature (positive or negative) of interchange energies are given in table 2. Structural details such as Bravais lattice, space group, number of atoms per unit cell, Pearson symbol, lattice parameters and co-ordinates of equivalent positions have been determined for each structure using International Tables for Crystallography (Hahn 1989). This information is recorded in table 3. However, this procedure does not yield any information for those structures for

Table 1. Energies of ground-state superstructures based on the hcp structure.

\begin{tabular}{ll}
\hline Phase & Energy \\
\hline$A_{12} B C$ & $\frac{3 N}{14}\left[W_{A B}^{1}+W_{C A}^{1}+W_{A B}^{2}+W_{C A}^{2}+W_{A B}^{3}+W_{C A}^{3}\right]$ \\
$A_{7} B_{6} C$ & $\frac{3 N}{14}\left[W_{B C}^{1}+6 W_{A B}^{2}+W_{C A}^{2}+6 W_{A B}^{3}+W_{C A}^{3}\right]$ \\
$A_{10} B C$ & $\frac{N}{4}\left[W_{A B}^{1}+W_{C A}^{1}+W_{A B}^{2}+W_{C A}^{2}+W_{A B}^{3}+W_{C A}^{3}\right]$ \\
$A_{6} B_{5} C$ & $\frac{N}{4}\left[W_{B C}^{1}+5 W_{A B}^{2}+W_{C A}^{2}+5 W_{A B}^{3}+W_{C A}^{3}\right]$ \\
$A_{5} B_{4} C$ & $\frac{3 N}{10}\left[W_{B C}^{1}+4 W_{A B}^{2}+W_{C A}^{2}+4 W_{A B}^{3}+W_{C A}^{3}\right]$ \\
$A_{6} B C$ & $\frac{3 N}{8}\left[W_{A B}^{1}+W_{C A}^{1}+W_{A B}^{2}+W_{C A}^{2}+W_{B C}^{3}\right]$ \\
$A_{4} B_{3} C$ & $\frac{3 N}{8}\left[W_{B C}^{1}+3 W_{A B}^{2}+W_{C A}^{2}+3 W_{A B}^{3}+W_{C A}^{3}\right]$ \\
$A_{7} B_{5} C_{2}$ & $\frac{3 N}{14}\left[2 W_{B C}^{1}+5 W_{A B}^{2}+2 W_{C A}^{2}+5 W_{A B}^{3}+2 W_{C A}^{3}\right]$ \\
$A_{3} B_{2} C$ & $\frac{N}{2}\left[W_{B C}^{1}+2 W_{A B}^{2}+W_{C A}^{2}+W_{A B}^{3}+W_{C A}^{3}\right]$ \\
\hline
\end{tabular}

Table 2. Nature of interchange energies for ordered ground-state structures.

\begin{tabular}{lccccccccc}
\hline & \multicolumn{7}{c}{ Sign of interchange energies } \\
\cline { 2 - 9 } Structure & $W_{A B}^{1}$ & $W_{B C}^{1}$ & $W_{C A}^{1}$ & $W_{A B}^{2}$ & $W_{B C}^{2}$ & $W_{C A}^{2}$ & $W_{A B}^{3}$ & $W_{B C}^{3}$ & $W_{C A}^{3}$ \\
\hline$A_{12} B C$ & - & + & - & - & + & - & - & + & - \\
$A_{7} B_{6} C$ & + & - & + & - & + & - & - & + & - \\
$A_{10} B C$ & - & + & - & - & + & - & - & + & - \\
$A_{6} B_{5} C$ & + & - & + & - & + & - & - & + & - \\
$A_{5} B_{4} C$ & + & - & + & - & + & - & - & + & - \\
$A_{6} B C$ & - & + & - & - & + & - & - & + & - \\
$A_{4} B_{3} C$ & + & - & + & - & + & - & - & + & - \\
$A_{7} B_{5} C_{2}$ & + & - & + & - & + & - & - & + & - \\
$A_{3} B_{2} C$ & + & - & + & - & + & - & - & + & - \\
\hline
\end{tabular}


Table 3. Crystallographic data for the ternary ground-state structures in hep alloys.

$A_{12} B C$ (I): Hexagonal

$P 6$ or $C_{3 k}^{1} ; 14$ atoms per unit cell

hP14

$a=a_{d}[310], b=a_{d}[320]$ and $c=c_{d}[001]$

Atomic positions:

$\begin{array}{lccccc} & \text { Wyckof } & & & & \\ \text { Atoms } & \text { notation } & \text { Symmetry } & x & y & z \\ C & 1(a) & 6 & 0 & 0 & 0 \\ B & 1(f) & 6 & 2 / 3 & 1 / 3 & 1 / 2 \\ A & 3(j) & m & 6 / 7 & 2 / 7 & 0 \\ A & 3(j) & m & 1 / 7 & 5 / 7 & 0 \\ A & 3(k) & m & 17 / 21 & 1 / 21 & 1 / 2 \\ A & 3(k) & m & 2 / 21 & 10 / 21 & 1 / 2\end{array}$

Site occupation probabilities: (figure $5 b$ )

$P_{C}^{5}=1, P_{B}^{8}=1, P_{A}^{1}=P_{A}^{2}=P_{A}^{3}=P_{A}^{4}=P_{A}^{6}=P_{A}^{7}=P_{A}^{9}=P_{A}^{10}=P_{A}^{11}=P_{A}^{12}=P_{A}^{13}=P_{A}^{14}=1$

$A_{12} B C$ (II): Monoclinic

$P m$ or $C_{s}^{1} ; 14$ atoms per unit cell

mP14

$a=a_{d}[110], b=a_{d}[430]$ and $c=c_{d}[001]$

$\alpha=\tan ^{-1}(-7 \sqrt{3} / 3)=103.9^{\prime}$

Atomic positions:

$\begin{array}{lccccc}\text { Atoms } & \text { Wyckof } & & & & \\ \text { notation } & \text { Symmetry } & x & y & z \\ B & 1(a) & m & 1 / 15 & 13 / 14 & 0 \\ A & 1(a) & m & 4 / 5 & 5 / 14 & 0 \\ A & 1(a) & m & 1 / 3 & 1 / 2 & 0 \\ A & 1(a) & m & 13 / 15 & 9 / 14 & 0 \\ A & 1(a) & m & 7 / 15 & 11 / 14 & 0 \\ A & 1(a) & m & 3 / 5 & 1 / 14 & 0 \\ A & 1(a) & m & 1 / 5 & 3 / 14 & 0 \\ A & 1(b) & m & 2 / 3 & 1 / 2 & 1 / 2 \\ A & 1(b) & m & 1 / 5 & 9 / 14 & 1 / 2 \\ A & 1(b) & m & 4 / 5 & 11 / 14 & 1 / 2 \\ A & 1(b) & m & 2 / 5 & 13 / 14 & 1 / 2 \\ A & 1(b) & m & 14 / 15 & 1 / 14 & 1 / 2 \\ A & 1(b) & m & 8 / 15 & 3 / 14 & 1 / 2 \\ A & 1(b) & m & 2 / 15 & 9 / 14 & 1 / 2\end{array}$

Site occupation probabilities: (figure 5 a)

$P_{C}^{S}=1, P_{B}^{1}=1, P_{A}^{2}=P_{A}^{3}=P_{A}^{4}=P_{A}^{6}=P_{A}^{7}=P_{A}^{8}=P_{A}^{9}=P_{A}^{10}=P_{A}^{11}=P_{A}^{12}=P_{A}^{13}=P_{A}^{14}=1$.

$A_{12} B C$ (III): Monoclinic

$P m$ or $C_{s}^{1}: 14$ atoms per unit cell $\mathrm{mP14}$

$a=a_{d}[\overline{110}], b=a_{d}[430]$ and $c=c_{d}[001]$

$\alpha=\tan ^{-1}(-7 \sqrt{3} / 3)=103.9^{\circ}$

Atomic positions:

$\begin{array}{lcclcl}\text { Atoms } & \begin{array}{c}\text { Wyckoff } \\ \text { notation }\end{array} & \text { Symmetry } & x & y & z \\ C & 1(a) & m & 1 / 5 & 13 / 14 & 0 \\ B & 1(a) & m & 1 / 3 & 1 / 2 & 0 \\ A & 1(a) & m & 4 / 5 & 5 / 14 & 0 \\ A & 1(a) & m & 13 / 15 & 9 / 14 & 0 \\ A & 1(a) & m & 7 / 15 & 11 / 14 & 0\end{array}$


Table 3 (Contd.)

\begin{tabular}{lllccc}
\hline$A$ & $1(a)$ & $m$ & $3 / 5$ & $1 / 14$ & 0 \\
$A$ & $1(a)$ & $m$ & $1 / 5$ & $3 / 14$ & 0 \\
$A$ & $1(b)$ & $m$ & $2 / 3$ & $1 / 2$ & $1 / 2$ \\
$A$ & $1(b)$ & $m$ & $1 / 5$ & $9 / 14$ & $1 / 2$ \\
$A$ & $1(b)$ & $m$ & $4 / 5$ & $11 / 14$ & $1 / 2$ \\
$A$ & $1(b)$ & $m$ & $2 / 5$ & $13 / 14$ & $1 / 2$ \\
$A$ & $1(b)$ & $m$ & $14 / 15$ & $1 / 14$ & $1 / 2$ \\
$A$ & $1(b)$ & $m$ & $8 / 15$ & $3 / 14$ & $1 / 2$ \\
$A$ & $1(b)$ & $m$ & $2 / 15$ & $9 / 14$ & $1 / 2$
\end{tabular}

Site occupation probabilities: (figure $5 \mathrm{a}$ )

$P_{C}^{5}=1, P_{B}^{2}=1, P_{A}^{1}=P_{A}^{3}=P_{A}^{4}=P_{A}^{6}=P_{A}^{7}=P_{A}^{8}=P_{A}^{9}=P_{A}^{10}=P_{A}^{11}=P_{A}^{12}=P_{A}^{13}=P_{A}^{14}=1$

$A_{12} B C$ (IV): Monoclinic

$P m$ or $C_{s}^{1} ; 14$ atoms per unit cell $\mathrm{mP14}$

$a=a_{d}[110], b=a_{d}[430]$ and $c=c_{d}[001]$

$\alpha=\tan ^{-1}(-7 \sqrt{3} / 3)=103.9^{\circ}$

Atomic positions:

$\begin{array}{lccccc}\text { Atoms } & \begin{array}{c}\text { Wyckoff } \\ \text { notation }\end{array} & \text { Symmetry } & x & y & z \\ C & 1(a) & m & 1 / 15 & 13 / 14 & 0 \\ B & 1(b) & m & 2 / 3 & 1 / 2 & 1 / 2 \\ A & 1(a) & m & 4 / 5 & 5 / 14 & 0 \\ A & 1(a) & m & 1 / 3 & 1 / 2 & 0 \\ A & 1(a) & m & 13 / 15 & 9 / 14 & 0 \\ A & 1(a) & m & 7 / 15 & 11 / 14 & 0 \\ A & 1(a) & m & 3 / 5 & 1 / 14 & 0 \\ A & 1(a) & m & 1 / 5 & 3 / 14 & 0 \\ A & 1(b) & m & 1 / 5 & 9 / 14 & 1 / 2 \\ A & 1(b) & m & 4 / 5 & 11 / 14 & 1 / 2 \\ A & 1(b) & m & 2 / 5 & 13 / 14 & 1 / 2 \\ A & 1(b) & m & 14 / 15 & 1 / 14 & 1 / 2 \\ A & 1(b) & m & 8 / 15 & 3 / 14 & 1 / 2 \\ A & 1(b) & m & 2 / 15 & 9 / 14 & 1 / 2\end{array}$

Site occupation probabilities: (figure $5 \mathrm{a}$ )

$P_{C}^{5}=1, P_{B}^{8}=1, P_{A}^{1}=P_{A}^{2}=P_{A}^{3}=P_{A}^{4}=P_{A}^{6}=P_{A}^{7}=P_{A}^{9}=P_{A}^{10}=P_{A}^{11}=P_{A}^{12}=P_{A}^{13}=P_{A}^{14}=1$

$A_{12} B C(V)$ : Monoclinic

$P m$ or $C_{s}^{1} ; 14$ atoms per unit cell mP14

$a=a_{d}[110], b=a_{d}[430]$ and $c=c_{d}[001]$

$\alpha=\tan ^{-1}(-7 \sqrt{3 / 3})=103.9^{\circ}$

Atomic positions:

$\begin{array}{lccccc}\text { Atoms } & \begin{array}{c}\text { Wyckoff } \\ \text { notation }\end{array} & \text { Symmetry } & x & y & z \\ C & 1(a) & m & 1 / 15 & 13 / 14 & 0 \\ B & 1(b) & m & 2 / 15 & 9 / 14 & 1 / 2 \\ A & 1(a) & m & 4 / 5 & 5 / 14 & 0 \\ A & 1(a) & m & 1 / 3 & 1 / 2 & 0 \\ A & 1(a) & m & 13 / 15 & 9 / 14 & 0 \\ A & 1(a) & m & 7 / 15 & 11 / 14 & 0 \\ A & 1(a) & m & 3 / 5 & 1 / 14 & 0 \\ A & 1(a) & m & 1 / 5 & 3 / 14 & 0 \\ A & 1(b) & m & 2 / 3 & 1 / 2 & 1 / 2\end{array}$


Table 3 (Contd.)

\begin{tabular}{lllccc}
\hline$A$ & $1(b)$ & $m$ & $1 / 5$ & $9 / 14$ & $1 / 2$ \\
$A$ & $1(b)$ & $m$ & $4 / 5$ & $11 / 14$ & $1 / 2$ \\
$A$ & $1(b)$ & $m$ & $2 / 5$ & $13 / 14$ & $1 / 2$ \\
$A$ & $1(b)$ & $m$ & $14 / 15$ & $1 / 14$ & $1 / 2$ \\
$A$ & $1(b)$ & $m$ & $8 / 15$ & $3 / 14$ & $1 / 2$
\end{tabular}

Site occupation probabilities: (figure 5a)

$P_{C}^{5}=1, P_{B}^{14}=1, P_{A}^{1}=P_{A}^{2}=P_{A}^{3}=P_{A}^{4}=P_{A}^{6}=P_{A}^{7}=P_{A}^{8}=P_{A}^{9}=P_{A}^{10}=P_{A}^{11}=P_{A}^{12}=P_{A}^{13}=1$

$A_{7} B_{6} C(I)$ : Hexagonal

$\mathrm{P} 6$ or $C_{3 h}^{1} ; 14$ atoms per unit cell

hP14

$a=a_{d}[310], b=a_{d}[320]$ and $c=c_{d}[001]$

Atomic positions:

$\begin{array}{lccccc}\text { Atoms } & \begin{array}{c}\text { Wyckof } \\ \text { notation }\end{array} & \text { Symmetry } & x & y & z \\ C & 1(a) & 6 & 0 & 0 & 0 \\ B & 3(j) & m & 6 / 7 & 2 / 7 & 0 \\ B & 3(j) & m & 1 / 7 & 5 / 7 & 0 \\ A & 1(f) & 6 & 2 / 3 & 1 / 3 & 1 / 2 \\ A & 3(k) & m & 17 / 21 & 1 / 21 & 1 / 2 \\ A & 3(k) & m & 2 / 21 & 10 / 21 & 1 / 2\end{array}$

Site occupation probabilities: (figure $5 b$ )

$P_{C}^{5}=1, P_{B}^{1}=P_{B}^{2}=P_{B}^{3}=P_{B}^{4}=P_{B}^{6}=P_{B}^{7}=1, P_{A}^{8}=P_{A}^{9}=P_{A}^{10}=P_{A}^{11}=P_{A}^{12}=P_{A}^{13}=P_{A}^{14}=1$

$A_{7} B_{6} C$ (II): Orthorhombic

$C 2 m m(A m m 2)$ or $C_{2 v}^{14} ; 28$ atoms per unit cell

oC28

$a=a_{d}[110], b=a_{d}[770]$ and $c=c_{d}[001]$

Atomic positions:

$\begin{array}{lccccc}\text { Atoms } & \text { Wyckof } & & & & \\ \text { notation } & \text { Symmetry } & x & y & z \\ C & 2(a) & 2 m m & 0 & 0 & 0 \\ B & 4(d) & m & 1 / 2 & 3 / 14 & 0 \\ B & 4(d) & m & 1 / 2 & 5 / 14 & 0 \\ B & 4(d) & m & 1 / 2 & 13 / 14 & 0 \\ A & 2(b) & 2 m m & 1 / 3 & 0 & 1 / 2 \\ A & 4(e) & m & 1 / 3 & 2 / 14 & 1 / 2 \\ A & 4(e) & m & 1 / 3 & 4 / 14 & 1 / 2 \\ A & 4(e) & m & 1 / 3 & 6 / 14 & 1 / 2\end{array}$

Site occupation probabilities: (figure 5 a)

$P_{C}^{5}=1, P_{B}^{1}=P_{B}^{2}=P_{B}^{3}=P_{B}^{4}=P_{B}^{6}=P_{B}^{7}=1, P_{A}^{8}=P_{A}^{9}=P_{A}^{10}=P_{A}^{11}=P_{A}^{12}=P_{A}^{13}=P_{A}^{14}=1$

$A_{10} B C$ (I): Orthorhombic

Pmm2 $(P 2 \mathrm{~mm})$ or $C_{2 v}^{1} ; 12$ atoms per unit cell

oP12

$a=a_{d}[110], b=a_{d}[330]$ and $c=c_{d}[001]$

Atomic positions:

$\begin{array}{lccccc}\text { Atoms } & \text { Wyckoff } & & & & \\ \text { notation } & \text { Symmetry } & x & y & z \\ B & 1(a) & 2 m m & 0 & 0 & 0 \\ A & 1(d) & 2 m m & 5 / 6 & 1 / 2 & 1 / 2 \\ A & 1(b) & 2 m m & 1 / 2 & 1 / 2 & 0 \\ & 1(c) & 2 m m & 1 / 3 & 0 & 1 / 2\end{array}$


Table 3 (Contd.)

\begin{tabular}{lllccc}
\hline$A$ & $2(g)$ & $m$ & $1 / 2$ & $1 / 6$ & 0 \\
$A$ & $2(g)$ & $m$ & 0 & $1 / 3$ & 0 \\
$A$ & $2(h)$ & $m$ & $1 / 3$ & $1 / 3$ & $1 / 2$ \\
$A$ & $2(h)$ & $m$ & $5 / 6$ & $1 / 6$ & $1 / 2$
\end{tabular}

Site occupation probabilities: (figure $4 a$ )

$P_{C}^{1}=1, P_{B}^{9}=1, P_{A}^{2}=P_{A}^{3}=P_{A}^{4}=P_{A}^{5}=P_{A}^{6}=P_{A}^{7}=P_{A}^{8}=P_{A}^{10}=P_{A}^{11}=P_{A}^{12}=1$

$A_{10} B C$ (II): Orthorhombic

Pmm2 (P2mm) or $C_{2 n}^{1} ; 12$ atoms per unit cell

oP12

$a=a_{d}[110], b=a_{d}[330]$ and $c=c_{d}[001]$

Atomic positions:

$\begin{array}{lccccc}\text { Atoms } & \text { Wyckoff } & & & & \\ \text { notation } & \text { Symmetry } & x & y & z \\ C & 1(a) & 2 m m & 0 & 0 & 0 \\ B & 1(b) & 2 m m & 1 / 2 & 1 / 2 & 0 \\ A & 1(c) & 2 m m & 1 / 3 & 0 & 1 / 2 \\ A & 1(d) & 2 m m & 5 / 6 & 1 / 2 & 1 / 2 \\ A & 2(g) & m & 1 / 2 & 1 / 6 & 0 \\ A & 2(g) & m & 0 & 1 / 3 & 0 \\ A & 2(h) & m & 1 / 3 & 1 / 3 & 1 / 2 \\ A & 2(h) & m & 5 / 6 & 1 / 6 & 1 / 2\end{array}$

Site occupation probabilities: (figure 4a)

$P_{C}^{1}=1, P_{B}^{4}=1, P_{A}^{2}=P_{A}^{3}=P_{A}^{5}=P_{A}^{6}=P_{A}^{7}=P_{A}^{8}=P_{A}^{9}=P_{A}^{10}=P_{A}^{11}=P_{A}^{12}=1$

$A_{10} B C$ (III): Monoclinic

$P m$ or $C_{s}^{1} ; 12$ atoms per unit cell

mP12

$a=a_{d}[120], b=a_{d}[220]$ and $c=c_{d}[001]$

$\alpha=\tan ^{-1}(-3 \sqrt{3})=100.9^{\circ}$

Atomic positions:

$\begin{array}{lccccc}\text { Atoms } & \begin{array}{c}\text { Wyckoff } \\ \text { notation }\end{array} & \text { Symmetry } & x & y & z \\ C & 1(a) & m & 0 & 0 & 0 \\ B & 1(b) & m & 5 / 9 & 11 / 18 & 1 / 2 \\ A & 1(a) & m & 1 / 3 & 1 / 6 & 0 \\ A & 1(a) & m & 2 / 3 & 1 / 3 & 0 \\ A & 1(a) & m & 0 & 1 / 2 & 0 \\ A & 1(a) & m & 1 / 3 & 2 / 3 & 0 \\ A & 1(a) & m & 2 / 3 & 5 / 6 & 0 \\ A & 1(b) & m & 8 / 9 & 5 / 18 & 1 / 2 \\ A & 1(b) & m & 2 / 9 & 8 / 18 & 1 / 2 \\ A & 1(b) & m & 8 / 9 & 5 / 18 & 1 / 2 \\ A & 1(b) & m & 2 / 9 & 8 / 18 & 1 / 2 \\ A & 1(b) & m & 5 / 9 & 2 / 18 & 1 / 2\end{array}$

Site occupation probabilities: (figure $4 b$ )

$P_{C}^{1}=1, P_{B}^{9}=1, P_{A}^{2}=P_{A}^{3}=P_{A}^{4}=P_{A}^{5}=P_{A}^{6}=P_{A}^{7}=P_{A}^{8}=P_{A}^{10}=P_{A}^{11}=P_{A}^{12}=1$

$A_{6} B_{5} C(I) ;$ Orthorhombic

$P 2 m m(P m m 2)$ or $C_{2 v}^{1} ; 12$ atoms per unit cell

oP12

$a=a_{d}[110], b=a_{d}[330]$ and $c=c_{d}[001]$ 
Table 3 (Contd.)

Atomic positions:

$\begin{array}{lccccc}\text { Atoms } & \text { Wyckoff } & & & & \\ \text { notation } & \text { Symmetry } & x & y & z \\ C & 1(a) & 2 m m & 0 & 0 & 0 \\ B & 1(b) & 2 m m & 1 / 2 & 1 / 2 & 0 \\ B & 2(g) & m & 1 / 2 & 1 / 6 & 0 \\ B & 2(g) & m & 0 & 1 / 3 & 0 \\ A & 1(c) & 2 m m & 1 / 3 & 0 & 1 / 2 \\ A & 1(d) & 2 m m & 5 / 6 & 1 / 2 & 1 / 2 \\ A & 2(h) & m & 1 / 3 & 1 / 3 & 1 / 2 \\ A & 2(h) & m & 5 / 6 & 1 / 6 & 1 / 2\end{array}$

Site occupation probabilities: (figure 4a)

$P_{C}^{1}=1, P_{B}^{2}=P_{B}^{3}=P_{B}^{4}=P_{B}^{S}=P_{B}^{6}=1, P_{A}^{7}=P_{A}^{8}=P_{A}^{9}=P_{A}^{10}=P_{A}^{11}=P_{A}^{12}=1$

$A_{6} B_{5} C$ (II): Monoclinic

$\mathrm{Pm}$ or $C_{s}^{1} ; 12$ atoms per unit cell $\mathrm{mP12}$

$a=a_{d}[120], b=a_{d}[220]$ and $c=c_{d}[001]$

$\alpha=\tan ^{-1}(-3 / \sqrt{3})=100.9^{\circ}$

Atomic positions:

$\begin{array}{lccccc}\text { Atoms } & \begin{array}{c}\text { Wyckof } \\ \text { notation }\end{array} & \text { Symmetry } & x & y & z \\ C & 1(a) & m & 0 & 0 & 0 \\ B & 1(a) & m & 1 / 3 & 1 / 6 & 0 \\ B & 1(a) & m & 2 / 3 & 1 / 3 & 0 \\ B & 1(a) & m & 0 & 1 / 2 & 0 \\ A & 1(a) & m & 1 / 3 & 2 / 3 & 0 \\ A & 1(a) & m & 2 / 3 & 5 / 6 & 0 \\ A & 1(b) & m & 8 / 9 & 5 / 18 & 1 / 2 \\ A & 1(b) & m & 2 / 9 & 8 / 18 & 1 / 2 \\ A & 1(b) & m & 8 / 9 & 14 / 18 & 1 / 2 \\ A & 1(b) & m & 2 / 9 & 17 / 18 & 1 / 2 \\ A & 1(b) & m & 5 / 9 & 2 / 18 & 1 / 2\end{array}$

Site occupation probabilities: (figure $4 b$ )

$P_{C}^{1}=1, P_{B}^{2}=P_{B}^{3}=P_{B}^{4}=P_{B}^{5}=P_{B}^{6}=1, P_{A}^{7}=P_{A}^{8}=P_{A}^{9}=P_{A}^{10}=P_{A}^{11}=P_{A}^{12}=1$

$A_{5} B_{4} C$ : Orthorhombic

$C 2 m m(A m m 2)$ or $C_{2 v}^{14} ; 20$ atoms per unit cell oC20

$a=a_{d}[110], b=a_{d}[550]$ and $c=c_{d}[001]$

Atomic positions:

$\begin{array}{lccccc}\text { Atoms } & \begin{array}{c}\text { Wyckof } \\ \text { notation }\end{array} & \text { Symmetry } & x & y & z \\ C & 2(a) & 2 m m & 1 / 2 & 0 & 0 \\ B & 4(d) & m & 1 / 2 & 2 / 10 & 0 \\ B & 4(d) & m & 1 / 2 & 6 / 10 & 0 \\ A & 2(b) & 2 m m & 5 / 6 & 0 & 1 / 2 \\ A & 4(e) & m & 1 / 3 & 1 / 10 & 1 / 2 \\ A & 4(e) & m & 5 / 6 & 2 / 10 & 1 / 2\end{array}$

Site occupation probabilities: (figure 3 )

$P_{C}^{1}=1, P_{B}^{2}=P_{B}^{3}=P_{B}^{4}=P_{B}^{S}=1, P_{A}^{6}=P_{A}^{7}=P_{A}^{8}=P_{A}^{9}=P_{A}^{10}=1$ 
Table 3 (Contd.)

$A_{6} B C$ : Hexagonal

$P{ }^{6} m 2$ or $D_{3 h}^{1} ; 8$ atoms per unit cell

hP8

$a=2 a_{d}[100], b=2 a_{d}[010]$ and $c=c_{d}[001]$

Atomic positions:

$\begin{array}{lccccc}\text { Atoms } & \begin{array}{c}\text { Wyckoff } \\ \text { notation }\end{array} & \text { Symmetry } & x & y & z \\ C & 1(a) & 6 m 2 & 0 & 0 & 0 \\ B & 1(e) & 6 m 2 & 2 / 3 & 1 / 3 & 1 / 2 \\ A & 3(j) & m m 2 & 1 / 2 & 1 / 2 & 0 \\ A & 3(k) & m m 2 & 1 / 6 & 5 / 6 & 1 / 2\end{array}$

Site occupation probabilities: (figure $2 \mathrm{~b}$ )

$P_{C}^{1}=1, P_{B}^{5}=1, P_{A}^{2}=P_{A}^{3}=P_{A}^{4}=P_{A}^{6}=P_{A}^{7}=P_{A}^{8}=1$

$A_{4} B_{3} C$ (I): Hexagonal

$P{ }^{6} \mathrm{~m} 2$ or $D_{3 h}^{1} ; 8$ atoms per unit cell

hP8

$a=2 a_{d}[100], b=2 a_{d}[010]$ and $c=c_{d}[001]$

Atomic positions:

$\begin{array}{lccccc}\text { Atoms } & \begin{array}{c}\text { Wyckoff } \\ \text { notation }\end{array} & \text { Symmetry } & \boldsymbol{x} & \boldsymbol{y} & z \\ C & 1(a) & 6 m 2 & 0 & 0 & 0 \\ B & 3(j) & m m 2 & 1 / 2 & 1 / 2 & 0 \\ A & 1(e) & 6 m 2 & 2 / 3 & 1 / 3 & 1 / 2 \\ A & 3(k) & m m 2 & 1 / 6 & 5 / 6 & 1 / 2\end{array}$

Site occupation probabilities: (figure $2 b$ )

$P_{C}^{1}=1, P_{B}^{2}=P_{B}^{3}=P_{B}^{4}=1, P_{A}^{5}=P_{A}^{6}=P_{A}^{7}=P_{A}^{8}=1$

$A_{4} B_{3} C$ (II): Orthorhombic

$P 2 m m(P m m 2)$ or $C_{2 v}^{1} ; 8$ atoms per unit cell

oP8

$a=a_{d}[110], b=a_{d}[220]$ and $c=c_{d}[001]$

Atomic positions:

$\begin{array}{lccccc}\text { Atoms } & \begin{array}{c}\text { Wyckoff } \\ \text { notation }\end{array} & \text { Symmetry } & x & y & z \\ C & 1(a) & 2 m m & 0 & 0 & 0 \\ B & 1(b) & 2 m m & 0 & 1 / 2 & 0 \\ B & 2(g) & m & 1 / 2 & 1 / 4 & 0 \\ A & 1(c) & 2 m m & 1 / 3 & 0 & 1 / 2 \\ A & 1(d) & 2 m m & 1 / 3 & 1 / 2 & 1 / 2 \\ A & 2(h) & m & 5 / 6 & 1 / 4 & 1 / 2\end{array}$

Site occupation probabilities: (figure 2a)

$P_{C}^{1}=1, P_{B}^{2}=P_{B}^{3}=P_{B}^{4}=1, P_{A}^{5}=P_{A}^{6}=P_{A}^{7}=P_{A}^{8}=1$

$A_{7} B_{5} C_{2}$ (I): Orthorhombic

$C 2 \mathrm{~mm}(A \mathrm{~mm} 2)$ or $C_{2 v}^{14} ; 28$ atoms per unit cell oC28

$a=a_{d}[110], b=a_{d}[770]$ and $c=c_{d}[001]$

Atomic positions:

$\begin{array}{lc}\text { Atoms } & \begin{array}{c}\text { Wyckoff } \\ \text { notation }\end{array} \\ C & 4(d) \\ B & 2(a)\end{array}$

Symmetry
$2 m$
$2 m m$

$x$
$1 / 2$
0

$y$
$5 / 14$
0


Table 3 (Contd.)

\begin{tabular}{lccccc}
\hline$B$ & $4(d)$ & $m$ & $1 / 2$ & $1 / 14$ & 0 \\
$B$ & $4(d)$ & $m$ & $1 / 2$ & $3 / 14$ & 0 \\
$A$ & $2(b)$ & $2 m m$ & $1 / 3$ & 0 & $1 / 2$ \\
$A$ & $4(e)$ & $m$ & $1 / 3$ & $2 / 14$ & $1 / 2$ \\
$A$ & $4(e)$ & $m$ & $5 / 6$ & $3 / 14$ & $1 / 2$ \\
$A$ & $4(e)$ & $m$ & $1 / 3$ & $6 / 14$ & $1 / 2$
\end{tabular}

Site occupation probabilities: (figure 5 a)

$P_{C}^{2}=P_{C}^{5}=1, P_{B}^{1}=P_{B}^{3}=P_{B}^{4}=P_{B}^{6}=P_{B}^{7}=1, P_{A}^{8}=P_{A}^{9}=P_{A}^{10}=P_{A}^{11}=P_{A}^{12}=P_{A}^{13}=P_{A}^{14}=1$

$A_{7} B_{5} C_{2}$ (II): Monoclinic

$P m$ or $C_{S}^{1} ; 14$ atoms per unit cell

$\mathrm{mP14}$

$a=a_{d}[110], b=a_{d}[430]$ and $c=c_{d}[001]$

$\alpha=\tan ^{-1}(-7 \sqrt{3} / 3)=103.9^{\circ}$

Atomic positions:

$\begin{array}{lccccc}\text { Atoms } & \begin{array}{c}\text { Wyckoff } \\ \text { notation }\end{array} & \text { Symmetry } & x & y & z \\ C & 1(a) & m & 4 / 5 & 5 / 14 & 0 \\ C & 1(a) & m & 1 / 15 & 13 / 14 & 0 \\ B & 1(a) & m & 1 / 3 & 1 / 2 & 0 \\ B & 1(a) & m & 13 / 15 & 9 / 14 & 0 \\ B & 1(a) & m & 7 / 15 & 11 / 14 & 0 \\ B & 1(a) & m & 3 / 5 & 1 / 14 & 0 \\ B & 1(a) & m & 1 / 5 & 3 / 14 & 0 \\ A & 1(b) & m & 2 / 3 & 1 / 2 & 1 / 2 \\ A & 1(b) & m & 1 / 5 & 9 / 14 & 1 / 2 \\ A & 1(b) & m & 4 / 5 & 11 / 14 & 1 / 2 \\ A & 1(b) & m & 2 / 5 & 13 / 14 & 1 / 2 \\ A & 1(b) & m & 14 / 15 & 1 / 14 & 1 / 2 \\ A & 1(b) & m & 8 / 15 & 3 / 14 & 1 / 2 \\ A & 1(b) & m & 2 / 15 & 9 / 14 & 1 / 2\end{array}$

Site occupation probabilities: (figure $5 \mathrm{a}$ )

$P_{C}^{1}=P_{C}^{5}=1, P_{B}^{2}=P_{B}^{3}=P_{B}^{4}=P_{B}^{6}=P_{B}^{7}=1, P_{A}^{8}=P_{A}^{9}=P_{A}^{10}=P_{A}^{11}=P_{A}^{12}=P_{A}^{13}=P_{A}^{14}=1$

$A_{3} B_{2} C$ : Hexagonal

$P \overline{6} 2 m$ or $D_{3 h}^{3} ; 6$ atoms per unit cell

hP6

$a=a_{d}[210], b=a_{d}[110]$ and $c=c_{d}[001]$

Atomic positions:

$\begin{array}{lccccc}\text { Atoms } & \text { Wyckof } & & & & \\ C & \text { notation } & \text { Symmetry } & x & y & z \\ B & 1(a) & 62 m & 0 & 0 & 0 \\ A & 2(c) & 6 & 1 / 3 & 2 / 3 & 0 \\ & 3(g) & m 2 m & 1 / 3 & 0 & 1 / 2\end{array}$

Site occupation probabilities: (figure 1) $P_{C}^{3}=1, P_{B}^{2}=P_{B}^{3}=1, P_{A}^{4}=P_{A}^{5}=P_{A}^{6}=1$

Other probabilities are zero.

which the values of different $q_{k}, r_{k}$ and $s_{k}$ lie between their maximum and minimum values. These structures may or may not be members of the ground-state family of hcp ternary alloys. We also observe that five structures with $A_{12} B C$ stoichiometry 
have the same energy but different structures. These structures are designated as $A_{12} B C$ (I), $A_{12} B C$ (II), $A_{12} B C$ (III), $A_{12} B C$ (IV) and $A_{12} B C(\mathrm{~V})$ and their crystallographic details are recorded in table 3 . A similar behaviour was observed for the structures $A_{7} B_{6} C, A_{10} B C, A_{6} B_{5} C, A_{4} B_{3} C$ and $A_{7} B_{5} C_{2}$ each of which is doubly degenerate. The complete crystallographic details about these degenerate structures are also given in table 3. This degeneracy can be eliminated by considering higher neighbour interactions.

\section{Ordered structures and their energies}

As mentioned earlier, ordered structures have also been generated by populating the atomic sites in case of division into eight sublattices with all possible combinations of $A, B$ and $C$ atoms (figure $2 \mathrm{~b}$ ). This results in five stoichiometries namely, $A_{6} B C, A_{5} B_{2} C, A_{4} B_{3} C, A_{4} B_{2} C_{2}$, and $A_{3} B_{3} C_{2}$ with $C_{A} \geqslant C_{B} \geqslant C_{C}$ while other stoichiometries can be obtained by interchanging $A, B$ and $C$ atoms. All the possible ordered structures for each stoichiometry have been enumerated and their energies evaluated. Some crystallographic information for these structures is given in tables 4-8. The procedure adopted is illustrated for the case of the $A_{6} B C^{2}$ structure in the following. In this structure, $C$ atoms are placed on sublattice $1, B$ on sublattice 2 and the remaining sublattices are populated by $A$ atoms. The [001] projection of the structure $A_{6} B C^{2}$ is shown in figure 6 . Now one can write the different $P_{\alpha}^{i}$ as follows:

$$
\begin{array}{ll}
P_{C}^{1}=1, P_{C}^{i}=0 & (i=2 \text { to } 8), \\
P_{B}^{1}=0, P_{B}^{2}=1, P_{B}^{i}=0 & (i=3 \text { to } 8) \\
P_{A}^{i}=0(i=1 \text { to } 2), P_{A}^{i}=1 & (i=3 \text { to } 8) .
\end{array}
$$

One can obtain the $q_{k}, r_{k}$ and $s_{k}$ (equations (5) to (7)) using the above probabilities and utilize these for the calculation of the configurational energy (equation (8)). Other structures with the same stoichiometry are obtained by taking $A, B$ and $C$

\begin{tabular}{|c|c|c|c|}
\hline $\begin{array}{l}\text { Ordered } \\
\text { structure } \\
\text { designation }\end{array}$ & $\begin{array}{l}\text { Space group* } \\
\text { Pearson } \\
\text { symbol }\end{array}$ & $\begin{array}{l}\text { Lattice } \\
\text { parameters** }\end{array}$ & Energy \\
\hline$A_{6} B C^{1}$ & $\begin{array}{l}P 6 m 2 \\
D \frac{1}{1 h} \\
h P 8\end{array}$ & $\begin{array}{l}a=2 a_{d}[100] \\
b=2 a_{d}[010] \\
c=c_{d}[001]\end{array}$ & $\begin{array}{l}(N / 8)\left[3 W_{A B}^{1}+3 W_{C A}^{1}+\right. \\
\left.3 W_{A B}^{2}+3 W_{C A}^{2}+3 W_{B C}^{3}\right]\end{array}$ \\
\hline \multicolumn{4}{|c|}{$P_{C}^{1}=1, P_{B}^{5}=1, P_{A}^{2}=P_{A}^{3}=P_{A}^{4}=P_{A}^{6}=P_{A}^{7}=P_{A}^{8}=1$} \\
\hline$A_{6} B C^{2}$ & $\begin{array}{l}C 2 m m(A m m 2) \\
C_{2 v}^{14} \\
o C 16\end{array}$ & $\begin{array}{l}a=2 a_{d}[210] \\
b=2 a_{d}[010] \\
c=c_{d}[001]\end{array}$ & $\begin{array}{l}(N / 8)\left[2 W_{A B}^{1}+W_{B C}^{1}+\right. \\
2 W_{C A}^{1}+3 W_{A B}^{2}+3 W_{C A}^{2}+ \\
\left.3 W_{A B}^{3}+3 W_{C A}^{3}\right]\end{array}$ \\
\hline \multicolumn{4}{|c|}{$P_{C}^{1}=1, P_{B}^{2}=1, P_{A}^{3}=P_{A}^{4}=P_{A}^{5}=P_{A}^{6}=P_{A}^{7}=P_{A}^{8}=1$} \\
\hline$A_{6} B C^{3}$ & $\begin{array}{l}C 2 m m(A m m 2) \\
C_{2 v}^{14} \\
o C 16\end{array}$ & $\begin{array}{l}a=2 a_{d}[210] \\
b=2 a_{d}[010] \\
c=c_{d}[001]\end{array}$ & $\begin{array}{l}(N / 8)\left[3 W_{A B}^{1}+3 W_{C A}^{1}+2 W_{A B}^{2}+\right. \\
\left.W_{B C}^{2}+2 W_{C A}^{2}+3 W_{A B}^{3}+3 W_{C A}^{3}\right]\end{array}$ \\
\hline$P_{C}^{1}=1, P_{B}^{6}=$ & $=P_{A}^{S}=P_{A}^{\gamma}=P_{A}^{8}=$ & & \\
\hline
\end{tabular}

Table 4. Crystal structure and energy of ordered superstructures with $A_{6} B C$ stoichiometry.

*International notation (standard orientation)/Schoenflies notation.

${ }^{* *} a_{d}$ and $c_{d}$ are lattice parameters of the disordered structure. 
Table 5. Crystal structure and energy of ordered superstructures with $A_{5} B_{2} C$ stoichiometry

\begin{tabular}{|c|c|c|c|}
\hline $\begin{array}{l}\text { Ordered } \\
\text { structure } \\
\text { designation }\end{array}$ & $\begin{array}{l}\text { Space group } \\
\text { Pearson } \\
\text { symbol }\end{array}$ & $\begin{array}{l}\text { Lattice } \\
\text { parameters }\end{array}$ & Energy \\
\hline$A_{5} B_{2} C^{1}$ & $\begin{array}{l}C 2 m m(A m m 2) \\
C_{20}^{14} \\
o C 16\end{array}$ & $\begin{array}{l}a=2 a_{d}[210] \\
b=2 a_{d}[010] \\
c=c_{d}[001]\end{array}$ & $\begin{array}{l}(N / 8)\left[5 W_{A B}^{1}+W_{B C}^{1}+\right. \\
2 W_{C A}^{1}+4 W_{A B}^{2}+3 W_{C A}^{2}+ \\
\left.3 W_{A B}^{3}+3 W_{B C}^{3}\right]\end{array}$ \\
\hline \multicolumn{4}{|c|}{$P_{C}^{1}=1, P_{B}^{2}=P_{B}^{5}=1, P_{A}^{3}=P_{A}^{4}=P_{A}^{6}=P_{A}^{7}=P_{A}^{8}=1$} \\
\hline$A_{5} B_{2} C^{2}$ & $\begin{array}{l}C 2 m m(A m m 2) \\
C_{2 v}^{14} \\
o C 16\end{array}$ & $\begin{array}{l}a=2 a_{d}[210] \\
b=2 a_{d}[010] \\
c=c_{d}[001]\end{array}$ & $\begin{array}{l}(N / 8)\left[5 W_{A B}^{1}+W_{B C}^{1}+\right. \\
2 W_{C A}^{1}+5 W_{A B}^{2}+W_{B C}^{2} \pm \\
\left.2 W_{C A}^{2}+3 W_{C A}^{3}\right]\end{array}$ \\
\hline \multicolumn{4}{|c|}{$P_{C}^{1}=1, P_{B}^{2}=P_{B}^{6}=1, P_{A}^{3}=P_{A}^{4}=P_{B}^{s}=P_{A}^{7}=P_{A}^{8}=1$} \\
\hline$A_{5} B_{2} C^{3}$ & $\begin{array}{l}C 2 m m(A m m 2) \\
C_{2 v}^{14} \\
o C 16\end{array}$ & $\begin{array}{l}a=a_{d}[220] \\
b=a_{d}[220] \\
c=c_{d}[001]\end{array}$ & $\begin{array}{l}(N / 8)\left[2 W_{A B}^{1}+2 W_{B C}^{1}+\right. \\
W_{C A}^{1}+6 W_{A B}^{2}+3 W_{C A}^{2}+ \\
\left.6 W_{A B}^{3}+3 W_{C A}^{3}\right]\end{array}$ \\
\hline \multicolumn{4}{|c|}{$P_{C}^{1}=1, P_{B}^{2}=P_{B}^{3}=1, P_{A}^{4}=P_{A}^{5}=P_{A}^{6}=P_{A}^{7}=P_{B}^{8}=1$} \\
\hline$A_{5} B_{2} C^{4}$ & $\begin{array}{l}P m \\
C_{s}^{1} \\
m P 8\end{array}$ & $\begin{array}{l}a=2 a_{d}[100] \\
b=2 a_{d}[010] \\
c=c_{d}[001]\end{array}$ & $\begin{array}{l}(N / 8)\left[5 W_{A B}^{1}+W_{B C}^{1}+\right. \\
2 W_{C A}^{1}+3 W_{A B}^{2}+W_{B C}^{2}+ \\
\left.2 W_{C A}^{2}+6 W_{A B}^{3}+3 W_{C A}^{3}\right]\end{array}$ \\
\hline \multicolumn{4}{|c|}{$P_{C}^{1}=1, P_{B}^{2}=P_{B}^{7}=1, P_{A}^{3}=P_{A}^{4}=P_{A}^{5}=P_{B}^{6}=P_{A}^{8}=1$} \\
\hline$A_{5} B_{2} C^{5}$ & $\begin{array}{l}C 2 m m(A m m 2) \\
C_{2 v}^{14} \\
o C 16\end{array}$ & $\begin{array}{l}a=2 a_{d}[210] \\
b=2 a_{d}[010] \\
c=c_{d}[001]\end{array}$ & $\begin{array}{l}(N / 8)\left[4 W_{A B}^{1}+3 W_{C A}^{1}+\right. \\
5 W_{A B}^{2}+W_{B C}^{2}+2 W_{C A}^{2}+ \\
\left.3 W_{A B}^{3}+3 W_{B C}^{3}\right]\end{array}$ \\
\hline \multicolumn{4}{|c|}{$P_{C}^{1}=1, P_{B}^{5}=P_{B}^{6}=1, P_{A}^{2}=P_{A}^{3}=P_{A}^{4}=P_{A}^{7}=P_{A}^{8}=1$} \\
\hline$A_{5} B_{2} C^{6}$ & $\begin{array}{l}C 2 m m(A m m 2) \\
C_{2 v}^{14} \\
o C 16\end{array}$ & $\begin{array}{l}a=a_{d}[220] \\
b=a_{d}[220] \\
c=c_{d}[001]\end{array}$ & $\begin{array}{l}(N / 8)\left[4 W_{A B}^{1}+3 W_{C A}^{1}+\right. \\
4 W_{A B}^{2}+2 W_{A C}^{2}+W_{C A}^{2}+ \\
\left.6 W_{A B}^{3}+3 W_{C A}^{3}\right]\end{array}$ \\
\hline \multicolumn{4}{|c|}{$P_{C}^{1}=1, P_{B}^{6}=P_{B}^{7}=1, P_{A}^{2}=P_{A}^{3}=P_{A}^{4}=P_{A}^{5}=P_{A}^{8}=1$} \\
\hline
\end{tabular}

atoms in the same proportions but populating the sublattices in all possible distinct ways. In a similar manner, all the structures for each of the stoichiometries listed earlier were obtained and the results are given in tables 4-8. We have arrived at three $A_{6} B C$, six $A_{5} B_{2} C$, eight $A_{4} B_{3} C$, sixteen $A_{4} B_{2} C_{2}$, and fifteen $A_{3} B_{3} C_{2}$ ordered structures in the present investigation. Of the 48 ordered structures, 15 are monoclinic, 29 orthorhombic and 4 hexagonal. Two structures namely $A_{6} B C^{1}$ and $A_{4} B_{3} C^{1}$ of the above list are ground state obtained by the procedure mentioned earlier.

\section{Discussion}

The ground-state structures $A_{12} B C, A_{7} B_{6} C, A_{10} B C, A_{6} B{ }_{5} C,{ }_{\mathrm{c}} A_{5} B_{4} C, A_{6} B C, A_{4} B_{3} C$, $A_{7} B_{5} C_{2}$ and $A_{3} B_{2} C$ can be obtained from 14 .(figures $5 \mathrm{a}$ and b), 14 (figures $5 \mathrm{a}$ and $b$ ), 12 (figures $4 a$ and $b$ ), 12 (figures $4 a$ and b), 10 (figure 3), 8 (figure 2b), 8 (figures $2 \mathrm{a}$ and $\mathrm{b}$ ), 14 (figures $5 \mathrm{a}$ and $\mathrm{b}$ ) and 12 (figure 4a) types of sublattice division respectively. The $A_{3} B_{2} C$ structure can also be generated by division into six (figure 1) sublattices.

The ground-state structures obtained in the present study (table 1) can also be 
Table 6. Crystal structure and energy of ordered superstructures with $A_{4} B_{3} C$ stoichiometry.

\begin{tabular}{|c|c|c|c|}
\hline $\begin{array}{l}\text { Ordered } \\
\text { structure } \\
\text { designation }\end{array}$ & $\begin{array}{l}\text { Space group } \\
\text { Pearson } \\
\text { symbol }\end{array}$ & $\begin{array}{l}\text { Lattice } \\
\text { parameters }\end{array}$ & Energy \\
\hline$A_{4} B_{3} C^{1}$ & $\begin{array}{l}P 6 m 2 \\
D ! \\
h P 8\end{array}$ & $\begin{array}{l}a=2 a_{d}[100] \\
b=2 a_{d}[010] \\
c=c_{d}[001]\end{array}$ & $\begin{array}{l}(N / 8)\left[3 W_{A C}^{1}+9 W_{A B}^{2}+\right. \\
\left.3 W_{C A}^{2}+9 W_{A B}^{3}+3 W_{C A}^{3}\right]\end{array}$ \\
\hline \multicolumn{4}{|c|}{$P_{C}^{1}=1, P_{B}^{2}=P_{B}^{3}=P_{B}^{4}=1, P_{A}^{5}=P_{A}^{6}=P_{A}^{7}=P_{A}^{8}=1$} \\
\hline$A_{4} B_{3} C^{2}$ & $\begin{array}{l}P m \\
C_{s}^{3} \\
m P 8\end{array}$ & $\begin{array}{l}a=2 a_{d}[100] \\
b=2 a_{d}[010] \\
c=c_{d}[001]\end{array}$ & $\begin{array}{l}(N / 8)\left[5 W_{A B}^{1}+2 W_{B C}^{1}+\right. \\
W_{C A}^{1}+6 W_{A B}^{2}+W_{B C}^{2}+ \\
\left.2 W_{C A}^{2}+3 W_{A B}^{3}+3 W_{C A}^{3}\right]\end{array}$ \\
\hline \multicolumn{4}{|c|}{$P_{C}^{1}=1, P_{B}^{2}=P_{B}^{3}=P_{B}^{6}=1, P_{A}^{4}=P_{A}^{5}=P_{A}^{7}=P_{A}^{8}=1$} \\
\hline$A_{4} B_{3} C^{3}$ & $\begin{array}{l}C 2 m m(A m m 2) \\
C_{2 v}^{14} \\
o C 16\end{array}$ & $\begin{array}{l}a=a_{d}[220] \\
b=a_{d}[220] \\
c=c_{d}[001]\end{array}$ & $\begin{array}{l}(N / 8)\left[6 W_{A B}^{1}+W_{B C}^{1}+\right. \\
2 W_{C A}^{1}+6 W_{A B}^{2}+W_{B C}^{2}+ \\
\left.2 W_{C A}^{2}+3 W_{B C}^{3}\right]\end{array}$ \\
\hline \multicolumn{4}{|c|}{$P_{C}^{1}=1, P_{B}^{4}=P_{B}^{5}=P_{B}^{8}=1, P_{A}^{2}=P_{A}^{3}=P_{A}^{6}=P_{A}^{7}=1$} \\
\hline$A_{4} B_{3} C^{4}$ & $\begin{array}{l}C 2 m m(A m m 2) \\
C_{2 x}^{14} \\
o C 16\end{array}$ & $\begin{array}{l}a=a_{d}[\overline{220}] \\
b=a_{d}[220] \\
c=c_{d}[001]\end{array}$ & $\begin{array}{l}(N / 8)\left[5 W_{A B}^{1}+2 W_{B C}^{1}+\right. \\
W_{C A}^{1}+5 W_{A B}^{2}+3 W_{C A}^{2}+ \\
\left.6 W_{A B}^{3}+3 W_{B C}^{3}\right]\end{array}$ \\
\hline \multicolumn{4}{|c|}{$P_{C}^{1}=1, P_{B}^{2}=P_{B}^{3}=P_{B}^{5}=1, P_{A}^{4}=P_{A}^{6}=P_{A}^{7}=P_{A}^{8}=1$} \\
\hline$A_{4} B_{3} C^{5}$ & $\begin{array}{l}C 2 m m(A m m 2) \\
C_{21}^{14} \\
o C 16\end{array}$ & $\begin{array}{l}a=a_{d}[\overline{220]} \\
b=a_{d}[220] \\
c=c_{d}[001]\end{array}$ & $\begin{array}{l}(N / 8)\left[5 W_{A B}^{1}+2 W_{B C}^{1}+\right. \\
W_{C A}^{1}+4 W_{A B}^{2}+W_{B C}^{2}+ \\
\left.2 W_{C A}^{2}+9 W_{A B}^{3}+3 W_{C A}^{3}\right]\end{array}$ \\
\hline \multicolumn{4}{|c|}{$P_{C}^{1}=1, P_{B}^{2}=P_{B}^{3}=P_{B}^{8}=1, P_{A}^{4}=P_{A}^{5}=P_{A}^{6}=P_{A}^{7}=1$} \\
\hline$A_{4} B_{3} C^{6}$ & $\begin{array}{l}P m \\
C_{S}^{1} \\
m P 8\end{array}$ & $\begin{array}{l}a=2 a_{d}[100] \\
b=2 a_{d}[010] \\
c=c_{d}[001]\end{array}$ & $\begin{array}{l}(N / 8)\left[6 W_{A B}^{1}+W_{B C}^{1}+\right. \\
2 W_{C A}^{1}+4 W_{A B}^{2}+W_{B C}^{2}+ \\
\left.2 W_{C A}^{2}+6 W_{A B}^{3}+3 W_{B C}^{3}\right]\end{array}$ \\
\hline \multicolumn{4}{|c|}{$P_{C}^{1}=1, P_{B}^{4}=P_{B}^{5}=P_{B}^{6}=1, P_{A}^{2}=\dot{P}_{A}^{3}=P_{A}^{7}=P_{A}^{8}=1$} \\
\hline$A_{4} B_{3} C^{7}$ & $\begin{array}{l}C 2 m m(A m m 2) \\
C_{2 v}^{14} \\
o C 16\end{array}$ & $\begin{array}{l}a=a_{d}[\mathbf{2 2 0 ]} \\
b=a_{d}[220] \\
c=c_{d}[001]\end{array}$ & $\begin{array}{l}(N / 8)\left[3 W_{A B}^{1}+3 W_{C A}^{1}+\right. \\
7 W_{A B}^{2}+2 W_{B C}^{2}+W_{C A}^{2}+ \\
\left.6 W_{A B}^{3}+3 W_{B C}^{3}\right]\end{array}$ \\
\hline \multicolumn{4}{|c|}{$P_{E}^{1}=1, P_{B}^{5}=P_{B}^{6}=P_{B}^{7}=1, P_{A}^{2}=P_{A}^{3}=P_{A}^{4}=P_{A}^{8}=1$} \\
\hline$A_{4} B_{3} C^{8}$ & $\begin{array}{l}P 6 m 2 \\
D_{3 h}^{1} \\
h P 8\end{array}$ & $\begin{array}{l}a=2 a_{d}[100] \\
b=2 a_{d}[010] \\
c=c_{d}[001]\end{array}$ & $\begin{array}{l}(N / 8)\left[3 W_{A B}^{1}+3 W_{C A}^{1}+\right. \\
6 W_{A B}^{2}+3 W_{B C}^{2}+ \\
\left.9 W_{A B}^{3}+3 W_{C A}^{3}\right]\end{array}$ \\
\hline$P_{C}^{1}=1, P_{B}^{6}=$ &,$P_{A}^{2}=P_{A}^{3}=P_{A}^{4}$ & & \\
\hline
\end{tabular}

generated by appropriate substitution of some $C$ atoms in binary ground-state structures identified in our earlier study using sublattice division method (Singh and Lele 1990). The $A_{7} B_{6} C, A_{6} B_{5} C, A_{5} B_{4} C$ and $A_{4} B_{3} C$ structures can be obtained by replacing one $B$ atom of $A B^{1}$ structure while $A_{7} B_{5} C_{2}$ and $A_{3} B_{2} C$ structures can be generated by replacing two $B$ atoms by $C$ atoms. The $A_{12} B C$ structure can be derived from a binary $A_{13} B$ structure. We have not considered this binary structure as ground state owing to entropy effects, but its energy is equal to the eniergy of a two-phase mixture of $A$ and $A_{5} B$ in an appropriate ratio. Similarly, the structure $A_{10} B C$ can be generated by appropriate substitution of $B$ atoms by $C$ atoms in the binary $A_{5} B$ structure. 
Table 7. Crystal structure and energy of ordered superstructures with $A_{4} B_{2} C_{2}$ stoichiometry.

\begin{tabular}{|c|c|c|c|}
\hline $\begin{array}{l}\text { Ordered } \\
\text { structure } \\
\text { designation }\end{array}$ & $\begin{array}{l}\text { Space group } \\
\text { Pearson } \\
\text { symbol }\end{array}$ & $\begin{array}{l}\text { Lattice } \\
\text { parameters }\end{array}$ & Energy \\
\hline$A_{4} B_{2} C_{2}^{1}$ & $\begin{array}{l}C 2 m m(A m m 2) \\
C_{2 v}^{14} \\
o C 16\end{array}$ & $\begin{array}{l}a=2 a_{d}[210] \\
b=2 a_{d}[010] \\
c=c_{d}[001]\end{array}$ & $\begin{array}{l}(N / 8)\left[4 W_{A B}^{1}+2 W_{B C}^{1}+\right. \\
2 W_{C A}^{1}+4 W_{A B}^{2}+2 W_{B C}^{2}+ \\
\left.4 W_{C A}^{2}+6 W_{C A}^{3}\right]\end{array}$ \\
\hline \multicolumn{4}{|c|}{$P_{C}^{1}=P_{C}^{2}=1, P_{B}^{3}=P_{B}^{7}=1, P_{A}^{4}=P_{A}^{5}=P_{A}^{6}=P_{A}^{8}=1$} \\
\hline$A_{4} B_{2} C_{2}^{2}$ & $\begin{array}{l}P 2 m m(P m m 2) \\
C_{2 v}^{1} \\
o P^{4}\end{array}$ & $\begin{array}{l}a=a_{d}[210] \\
b=a_{d}[010] \\
c=c_{d}[001]\end{array}$ & $\begin{array}{l}(N / 8)\left[4 W_{A B}^{1}+4 W_{C A}^{1}+\right. \\
4 W_{A B}^{2}+2 W_{B C}^{2}+ \\
\left.4 W_{C A}^{2}+6 W_{B C}^{3}\right]\end{array}$ \\
\hline \multicolumn{4}{|c|}{$P_{C}^{1}=P_{C}^{2}=1, P_{B}^{5}=P_{B}^{6}=1, P_{A}^{3}=P_{A}^{4}=P_{A}^{7}=P_{A}^{8}=1$} \\
\hline$A_{4} B_{2} C_{2}^{3}$ & $\begin{array}{l}\text { Cmcm } \\
D_{2 h}^{17} \\
o C 16\end{array}$ & $\begin{array}{l}a=2 a_{d}[210] \\
b=2 a_{d}[010] \\
c=c_{d}[001]\end{array}$ & $\begin{array}{l}(N / 8)\left[4 W_{A B}^{1}+2 W_{B C}^{1}+\right. \\
4 W_{C A}^{1}+4 W_{A B}^{2}+ \\
\left.2 W_{B C}^{2}+4 W_{C A}^{2}\right]\end{array}$ \\
\hline \multicolumn{4}{|c|}{$P_{C}^{1}=P_{C}^{5}=1, P_{B}^{2}=P_{B}^{6}=1, P_{A}^{7}=P_{A}^{4}=P_{A}^{7}=P_{A}^{8}=1$} \\
\hline$A_{4} B_{2} C_{2}^{4}$ & $\begin{array}{l}P 2 m m(P m m 2) \\
C_{2 v}^{1} \\
o P 4\end{array}$ & $\begin{array}{l}a=a_{d}[210] \\
b=a_{d}[010] \\
c=c_{d}[001]\end{array}$ & $\begin{array}{l}(N / 8)\left[4 W_{B C}^{1}+6 W_{A B}^{2}+\right. \\
\left.6 W_{C A}^{2}+6 W_{A B}^{3}+6 W_{C A}^{3}\right]\end{array}$ \\
\hline \multicolumn{4}{|c|}{$P_{C}^{1}=P_{C}^{2}=1, P_{B}^{3}=P_{B}^{4}=1, P_{A}^{5}=P_{A}^{6}=P_{A}^{7}=P_{A}^{8}=1$} \\
\hline$A_{4} B_{2} C_{2}^{5}$ & $\begin{array}{l}P m \\
C_{s}^{1} \\
m P 8\end{array}$ & $\begin{array}{l}a=2 a_{d}[100] \\
b=2 a_{d}[010] \\
c=c_{d}[001]\end{array}$ & $\begin{array}{l}(N / 8)\left[4 W_{A B}^{1}+2 W_{B C}^{1}+\right. \\
2 W_{C A}^{1}+3 W_{A B}^{2}+W_{B C}^{2}+5 W_{C A}^{2}+ \\
\left.3 W_{A B}^{3}+3 W_{B C}^{3}+3 W_{C A}^{3}\right]\end{array}$ \\
\hline \multicolumn{4}{|c|}{$P_{C}^{1}=P_{C}^{2}=1, P_{B}^{3}=P_{B}^{5}=1, P_{A}^{4}=P_{A}^{6}=P_{A}^{7}=P_{A}^{8}=1$} \\
\hline$A_{4} B_{2} C_{2}^{6}$ & $\begin{array}{l}C 2 m m(A m m 2) \\
C_{2 v}^{14} \\
o C 16\end{array}$ & $\begin{array}{l}a=2 a_{d}[210] \\
b=2 a_{d}[010] \\
c=c_{d}[001]\end{array}$ & $\begin{array}{l}(N / 8)\left[4 W_{A B}^{1}+2 W_{B C}^{1}+\right. \\
4 W_{C A}^{1}+2 W_{A B}^{2}+2 W_{B C}^{2}+ \\
\left.4 W_{C A}^{2}+6 W_{A B}^{3}+6 W_{C A}^{3}\right]\end{array}$ \\
\hline \multicolumn{4}{|c|}{$P_{C}^{1}=P_{C}^{2}=1, P_{B}^{3}=P_{B}^{8}=1, P_{A}^{4}=P_{A}^{5}=P_{A}^{6}=P_{A}^{7}=1$} \\
\hline$A_{4} B_{2} C_{2}^{7}$ & $\begin{array}{l}P m \\
C_{s}^{1} \\
m P 8\end{array}$ & $\begin{array}{l}a=2 a_{d}[100] \\
b=2 a_{d}[010] \\
c=c_{d}[001]\end{array}$ & $\begin{array}{l}(N / 8)\left[4 W_{A B}^{1}+4 W_{C A}^{1}+\right. \\
3 W_{A B}^{2}+3 W_{B C}^{2}+3 W_{C A}^{2}+ \\
\left.3 W_{A B}^{3}+3 W_{B C}^{3}+3 W_{C A}^{3}\right]\end{array}$ \\
\hline \multicolumn{4}{|c|}{$P_{C}^{1}=P_{C}^{2}=1, P_{B}^{5}=P_{B}^{7}=1, P_{A}^{3}=P_{A}^{4}=P_{A}^{6}=P_{A}^{8}=1$} \\
\hline$A_{4} B_{2} C_{2}^{8}$ & $\begin{array}{l}P 2 m m(P m m 2) \\
C_{2 v}^{1} \\
o P 4\end{array}$ & $\begin{array}{l}a=a_{d}[210] \\
b=a_{d}[010] \\
c=c_{d}[001]\end{array}$ & $\begin{array}{l}(N / 8)\left[4 W_{A B}^{1}+4 W_{C A}^{1}+\right. \\
2 W_{A B}^{2}+4 W_{B C}^{2}+2 W_{C A}^{2}+ \\
\left.6 W_{A B}^{3}+6 W_{C A}^{3}\right]\end{array}$ \\
\hline \multicolumn{4}{|c|}{$P_{C}^{1}=P_{C}^{2}=1, P_{B}^{7}=P_{B}^{8}=1, P_{A}^{3}=P_{A}^{4}=P_{A}^{5}=P_{A}^{6}=1$} \\
\hline$A_{4} B_{2} C_{2}^{9}$ & $\begin{array}{l}C 2 m m(A m m 2) \\
C_{20}^{14} \\
o C 16\end{array}$ & $\begin{array}{l}a=a_{d}[220] \\
b=a_{d}[220] \\
c=c_{d}[001]\end{array}$ & $\begin{array}{l}(N / 8)\left[2 W_{A B}^{1}+2 W_{B C}^{1}+\right. \\
4 W_{C A}^{1}+4 W_{A B}^{2}+2 W_{B C}^{2}+ \\
\left.4 W_{C A}^{2}+6 W_{A B}^{3}\right]\end{array}$ \\
\hline \multicolumn{4}{|c|}{$P_{C}^{1}=P_{C}^{5}=1, P_{B}^{2}=P_{B}^{3}=1, P_{A}^{4}=P_{A}^{6}=P_{A}^{7}=P_{A}^{8}=1$} \\
\hline$A_{4} B_{2} C_{2}^{10}$ & $\begin{array}{l}C c 2 m(A m a 2) \\
C_{2 v}^{16} \\
o C 16\end{array}$ & $\begin{array}{l}a=a_{d}[220] \\
b=a_{d}[220] \\
c=c_{d}[001]\end{array}$ & $\begin{array}{l}(N / 8)\left[4 W_{A B}^{1}+2 W_{B C}^{1}+\right. \\
4 W_{C A}^{1}+2 W_{A B}^{2}+2 W_{B C}^{2}+ \\
\left.4 W_{C A}^{2}+6 W_{A B}^{3}\right]\end{array}$ \\
\hline \multicolumn{4}{|c|}{$P_{C}^{1}=P_{C}^{5}=1, P_{B}^{2}=P_{B}^{7}=1, P_{A}^{3}=P_{A}^{4}=P_{A}^{6}=P_{A}^{8}=1$} \\
\hline$A_{4} B_{2} C_{2}^{11}$ & $\begin{array}{l}P m \\
C_{s}^{1} \\
m P 8\end{array}$ & $\begin{array}{l}a=2 a_{d}[100] \\
b=2 a_{d}[010] \\
c=c_{d}[001]\end{array}$ & $\begin{array}{l}(N / 8)\left[2 W_{A B}^{1}+2 W_{B C}^{1}+\right. \\
4 W_{C A}^{1}+5 W_{A B}^{2}+W_{B C}^{2}+ \\
3 W_{C A}^{2}+3 W_{A B}^{3}+ \\
\left.3 W_{B C}^{3}+3 W_{C A}^{3}\right]\end{array}$ \\
\hline
\end{tabular}


Table 7 (Contd.)

\begin{tabular}{|c|c|c|c|}
\hline$A_{4} B_{2} C_{2}^{12}$ & $\begin{array}{l}\text { Ccmm }(\mathrm{Cmcm}) \\
D_{2 h}^{17} \\
o C 16\end{array}$ & $\begin{array}{l}a=2 a_{d}[210] \\
b=2 a_{d}[010] \\
c=c_{d}[001]\end{array}$ & $\begin{array}{l}(N / 8)\left[4 W_{A B}^{1}+2 W_{B C}^{1}+\right. \\
4 W_{C A}^{1}+4 W_{A B}^{2}+ \\
\left.4 W_{C A}^{2}+6 W_{B C}^{3}\right]\end{array}$ \\
\hline \multicolumn{4}{|c|}{$P_{C}^{1}=P_{C}^{6}=1, P_{B}^{2}=P_{B}^{5}=1, P_{A}^{3}=P_{A}^{4}=P_{A}^{7}=P_{A}^{8}=1$} \\
\hline$A_{4} B_{2} C_{2}^{13}$ & $\begin{array}{l}P m \\
C_{s}^{1} \\
m P 8\end{array}$ & $\begin{array}{l}a=2 a_{d}[100] \\
b=2 a_{d}[010] \\
c=c_{d}[001]\end{array}$ & $\begin{array}{l}(N / 8)\left[4 W_{A B}^{1}+2 W_{B C}^{1}+4 W_{C A}^{1}+\right. \\
3 W_{A B}^{2}+W_{B C}^{2}+3 W_{C A}^{2}+ \\
\left.3 W_{A B}^{3}+3 W_{B C}^{3}+3 W_{C A}^{3}\right]\end{array}$ \\
\hline \multicolumn{4}{|c|}{$P_{C}^{1}=P_{C}^{6}=1, P_{B}^{2}=P_{B}^{7}=1, P_{A}^{3}=P_{A}^{4}=P_{A}^{5}=P_{A}^{8}=1$} \\
\hline$A_{4} B_{2} C_{2}^{14}$ & $\begin{array}{l}C 2 m m(A m m 2) \\
C_{2 v}^{14} \\
o C 16\end{array}$ & $\begin{array}{l}a=2 a_{d}[210] \\
b=2 a_{d}[010] \\
c=c_{d}[001]\end{array}$ & $\begin{array}{l}(N / 8)\left[2 W_{A B}^{1}+2 W_{B C}^{1}+\right. \\
4 W_{C A}^{1}+4 W_{A B}^{2}+2 W_{B C}^{2}+ \\
\left.2 W_{C A}^{2}+6 W_{A B}^{3}+6 W_{C A}^{3}\right]\end{array}$ \\
\hline \multicolumn{4}{|c|}{$P_{C}^{1}=P_{C}^{6}=1, P_{B}^{3}=P_{B}^{4}=1, P_{A}^{2}=P_{A}^{5}=P_{A}^{7}=P_{A}^{8}=1$} \\
\hline$A_{4} B_{2} C_{2}^{15}$ & $\begin{array}{l}C c 2 m(A m a 2) \\
C_{20}^{16} \\
o C 16\end{array}$ & $\begin{array}{l}a=2 a_{d}[210] \\
b=2 a_{d}[010] \\
c=c_{d}[001]\end{array}$ & $\begin{array}{l}(N / 8)\left[4 W_{A B}^{1}+2 W_{B C}^{1}+\right. \\
4 W_{C A}^{1}+4 W_{A B}^{2}+2 W_{B C}^{2}+ \\
\left.3 W_{C A}^{2}+6 W_{C A}^{3}\right]\end{array}$ \\
\hline \multicolumn{4}{|c|}{$P_{C}^{1}=P_{C}^{6}=1, P_{B}^{3}=P_{B}^{7}=1, P_{A}^{2}=P_{A}^{4}=P_{A}^{5}=P_{A}^{8}=1$} \\
\hline$A_{4} B_{2} C_{2}^{16}$ & $\begin{array}{l}P m \\
C_{s}^{1} \\
m P 8\end{array}$ & $\begin{array}{l}a=2 a_{d}[100] \\
b=2 a_{d}[010] \\
c=c_{d}[001]\end{array}$ & $\begin{array}{l}(N / 8)\left[4 W_{A B}^{1}+2 W_{B C}^{1}+\right. \\
4 W_{C A}^{1}+2 W_{A B}^{2}+2 W_{B C}^{2}+ \\
\left.2 W_{C A}^{2}+6 W_{A B}^{3}+6 W_{C A}^{3}\right]\end{array}$ \\
\hline$P_{C}^{1}=P_{C}^{6}=$ & $P_{A}^{2}=P_{A}^{4}=P_{A}^{5}=$ & & \\
\hline
\end{tabular}

Table 8. Crystal structure and energy of ordered superstructures with $A_{3} B_{3} C_{2}$ stoichiometry.

\begin{tabular}{|c|c|c|c|}
\hline $\begin{array}{l}\text { Ordered } \\
\text { structure } \\
\text { designation }\end{array}$ & $\begin{array}{l}\text { Space group } \\
\text { Pearson } \\
\text { symbol }\end{array}$ & $\begin{array}{l}\text { Lattice } \\
\text { parameters }\end{array}$ & Energy \\
\hline$A_{3} B_{3} C_{2}^{1}$ & $\begin{array}{l}C 2 m m(A m m 2) \\
C \frac{14}{2 v} \\
o C 16\end{array}$ & $\begin{array}{l}a=2 a_{d}[210] \\
b=2 a_{d}[010] \\
c=c_{d}[001]\end{array}$ & $\begin{array}{l}(N / 8)\left[3 W_{A B}^{1}+4 W_{B C}^{1}+\right. \\
5 W_{A B}^{2}+2 W_{B C}^{2}+4 W_{C A}^{2}+ \\
\left.3 W_{A B}^{3}+6 W_{C A}^{3}\right]\end{array}$ \\
\hline \multicolumn{4}{|c|}{$P_{C}^{1}=P_{C}^{2}=1, P_{B}^{3}=P_{B}^{4}=P_{B}^{7}=1, P_{A}^{5}=P_{A}^{6}=P_{A}^{8}=1$} \\
\hline$A_{3} B_{3} C_{2}^{2}$ & $\begin{array}{l}C 2 m m(A m m 2) \\
C_{2 v}^{14} \\
o C 16\end{array}$ & $\begin{array}{l}a=2 a_{d}[210] \\
b=2 a_{d}[010] \\
c=c_{d}[001]\end{array}$ & $\begin{array}{l}(N / 8)\left[5 W_{A B}^{1}+2 W_{B C}^{1}+\right. \\
2 W_{C A}^{1}+3 W_{A B}^{2}+2 W_{B C}^{2}+ \\
\left.4 W_{C A}^{2}+3 W_{A B}^{3}+6 W_{B C}^{3}\right]\end{array}$ \\
\hline \multicolumn{4}{|c|}{$P_{C}^{1}=P_{C}^{2}=1, P_{B}^{4}=P_{B}^{5}=P_{B}^{6}=1, P_{A}^{3}=P_{A}^{7}=P_{A}^{8}=1$} \\
\hline$A_{3} B_{3} C_{2}^{3}$ & $\begin{array}{l}P m \\
C_{s}^{1} \\
m P 8\end{array}$ & $\begin{array}{l}a=2 a_{d}[100] \\
b=2 a_{d}[010] \\
c=c_{d}[001]\end{array}$ & $\begin{array}{l}(N / 8)\left[5 W_{A B}^{1}+2 W_{B C}^{1}+\right. \\
2 W_{C A}^{1}+4 W_{A B}^{2}+3 W_{B C}^{2}+ \\
\left.3 W_{C A}^{2}+3 W_{B C}^{3}+3 W_{C A}^{3}\right]\end{array}$ \\
\hline \multicolumn{4}{|c|}{$P_{C}^{1}=P_{C}^{2}=1, P_{B}^{4}=P_{B}^{5}=P_{B}^{8}=1, P_{A}^{3}=P_{A}^{6}=P_{A}^{7}=1$} \\
\hline$A_{3} B_{3} C_{2}^{4}$ & $\begin{array}{l}P m \\
C_{s}^{1} \\
m P 8\end{array}$ & $\begin{array}{l}a=2 a_{d}[100] \\
b=2 a_{d}[010] \\
c=c_{d}[001]\end{array}$ & $\begin{array}{l}(N / 8)\left[4 W_{A B}^{1}+3 W_{B C}^{1}+\right. \\
3 W_{C A}^{1}+4 W_{A B}^{2}+3 W_{B C}^{2}+ \\
\left.3 W_{C A}^{2}+3 W_{A B}^{3}\right]\end{array}$ \\
\hline \multicolumn{4}{|c|}{$P_{C}^{1}=P_{C}^{5}=1, P_{B}^{2}=P_{B}^{3}=P_{B}^{6}=1, P_{A}^{4}=P_{A}^{7}=P_{A}^{8}=1$} \\
\hline$A_{3} B_{3} C_{2}^{5}$ & $\begin{array}{l}C 2 m m(A m m 2) \\
C_{2 v}^{14} \\
o C 16\end{array}$ & $\begin{array}{l}a=2 a_{d}[210] \\
b=2 a_{d}[010] \\
c=c_{d}[001]\end{array}$ & $\begin{array}{l}(N / 8)\left[3 W_{A B}^{1}+4 W_{B C}^{1}+\right. \\
4 W_{A B}^{2}+W_{B C}^{2}+5 W_{C A}^{2}+ \\
\left.6 W_{A B}^{3}+3 W_{B C}^{3}+3 W_{C A}^{3}\right]\end{array}$ \\
\hline
\end{tabular}


Table 8 (Contd)

\begin{tabular}{|c|c|c|c|}
\hline $\begin{array}{l}\text { Ordered } \\
\text { structure } \\
\text { designation }\end{array}$ & $\begin{array}{l}\text { Space group } \\
\text { Pearson } \\
\text { symbol }\end{array}$ & $\begin{array}{l}\text { Lattice } \\
\text { parameters }\end{array}$ & Energy \\
\hline$A_{3} B_{3} C_{2}^{6}$ & $\begin{array}{l}P m \\
C_{3}^{1} \\
m P 8\end{array}$ & $\begin{array}{l}a=2 a_{d}[100] \\
b=2 a_{d}[010] \\
c=c_{d}[001]\end{array}$ & $\begin{array}{l}(N / 8)\left[5 W_{A B}^{1}+2 W_{B C}^{1}+\right. \\
2 W_{C A}^{1}+2 W_{A B}^{2}+3 W_{B C}^{2}+3 W_{C A}^{2}+ \\
\left.6 W_{A B}^{3}+3 W_{B C}^{3}+3 W_{C A}^{3}\right]\end{array}$ \\
\hline \multicolumn{4}{|c|}{$P_{C}^{1}=P_{C}^{2}=1, P_{B}^{4}=P_{B}^{5}=P_{B}^{7}=1, P_{A}^{3}=P_{A}^{6}=P_{A}^{8}=1$} \\
\hline$A_{3} B_{3} C_{2}^{7}$ & $\begin{array}{l}C 2 m m(A m m 2) \\
C_{2 v}^{14} \\
o C 16\end{array}$ & $\begin{array}{l}a=2 a_{d}[210] \\
b=2 a_{d}[010] \\
c=c_{d}[001]\end{array}$ & $\begin{array}{l}(N / 8)\left[3 W_{A B}^{1}+4 W_{C A}^{1}+\right. \\
5 W_{A B}^{2}+4 W_{B C}^{2}+2 W_{C A}^{2}+ \\
\left.3 W_{A B}^{3}+6 W_{B C}^{3}\right]\end{array}$ \\
\hline \multicolumn{4}{|c|}{$P_{C}^{1}=P_{C}^{2}=1, P_{B}^{5}=P_{B}^{6}=P_{B}^{7}=1, P_{A}^{3}=P_{A}^{4}=P_{A}^{8}=1$} \\
\hline$A_{3} B_{3} C_{2}^{8}$ & $\begin{array}{l}C 2 \mathrm{~mm}(A m m 2) \\
C_{2 v}^{14} \\
o C 16\end{array}$ & $\begin{array}{l}a=2 a_{d}[210] \\
b=2 a_{d}[010] \\
c=c_{d}[001]\end{array}$ & $\begin{array}{l}(N / 8)\left[3 W_{A B}^{1}+4 W_{C A}^{1}+\right. \\
4 W_{A B}^{2}+5 W_{B C}^{2}+W_{C A}^{2}+ \\
\left.6 W_{A B}^{3}+3 W_{B C}^{3}+3 W_{C A}^{3}\right]\end{array}$ \\
\hline \multicolumn{4}{|c|}{$P_{C}^{1}=P_{C}^{2}=1, P_{B}^{6}=P_{B}^{7}=P_{B}^{8}=1, P_{A}^{3}=P_{A}^{4}=P_{A}^{3}=1$} \\
\hline$A_{3} B_{3} C_{2}^{9}$ & $\begin{array}{l}P \not{6} m 2 \\
D_{3 h}^{1} \\
h P 8\end{array}$ & $\begin{array}{l}a=2 a_{d}[100] \\
b=2 a_{d}[010] \\
c=c_{d}[001]\end{array}$ & $\begin{array}{l}(N / 8)\left[3 W_{B C}^{1}+3 W_{C A}^{1}+6 W_{A B}^{2}+\right. \\
\left.3 W_{B C}^{2}+3 W_{C A}^{2}+9 W_{A B}^{3}\right]\end{array}$ \\
\hline \multicolumn{4}{|c|}{$P_{C}^{1}=P_{C}^{5}=1, P_{B}^{2}=P_{B}^{3}=P_{B}^{4}=1, P_{A}^{6}=P_{A}^{7}=P_{A}^{8}=1$} \\
\hline$A_{3} B_{3} C_{2}^{10}$ & $\begin{array}{l}C 2 m m(A m m 2) \\
C_{2 v}^{14} \\
o C_{16}\end{array}$ & $\begin{array}{l}a=a_{d}[220] \\
b=a_{d}[220] \\
c=c_{d}[001]\end{array}$ & $\begin{array}{l}(N / 8)\left[4 W_{A B}^{1}+3 W_{B C}^{1}+\right. \\
3 W_{C A}^{1}+2 W_{A B}^{2}+3 W_{B C}^{2}+ \\
\left.3 W_{C A}^{2}+9 W_{A B}^{3}\right]\end{array}$ \\
\hline \multicolumn{4}{|c|}{$P_{C}^{1}=P_{C}^{5}=1, P_{B}^{2}=P_{B}^{3}=P_{B}^{8}=1, P_{A}^{4}=P_{A}^{6}=P_{A}^{7}=1$} \\
\hline$A_{3} B_{3} C_{2}^{11}$ & $\begin{array}{l}C 2 m m(A m m 2) \\
C_{2 v}^{14} \\
o C 16\end{array}$ & $\begin{array}{l}a=2 a_{d}[210] \\
b=2 a_{d}[010] \\
c=c_{d}[001]\end{array}$ & $\begin{array}{l}(N / 8)\left[3 W_{B C}^{1}+3 W_{C A}^{1}+\right. \\
7 W_{A B}^{2}+2 W_{B C}^{2}+2 W_{C A}^{2}+ \\
\left.6 W_{A B}^{3}+3 W_{B C}^{3}+3 W_{C A}^{3}\right]\end{array}$ \\
\hline \multicolumn{4}{|c|}{$P_{C}^{1}=P_{C}^{6}=1, P_{B}^{2}=P_{B}^{3}=P_{B}^{4}=1, P_{A}^{5}=P_{A}^{7}=P_{A}^{8}=1$} \\
\hline$A_{3} B_{3} C_{2}^{12}$ & $\begin{array}{l}P m \\
C_{s}^{1} \\
m P 8\end{array}$ & $\begin{array}{l}a=2 a_{d}[100] \\
b=2 a_{d}[010] \\
c=c_{d}[001]\end{array}$ & $\begin{array}{l}(N / 8)\left[4 W_{A B}^{1}+3 W_{B C}^{1}+\right. \\
3 W_{C A}^{1}+4 W_{A B}^{2}+W_{B C}^{2}+ \\
\left.3 W_{C A}^{2}+3 W_{A B}^{3}+6 W_{B C}^{3}\right]\end{array}$ \\
\hline \multicolumn{4}{|c|}{$P_{C}^{1}=P_{C}^{6}=1, P_{B}^{2}=P_{B}^{3}=P_{B}^{5}=1, P_{A}^{4}=P_{A}^{7}=P_{A}^{8}=1$} \\
\hline$A_{3} B_{3} C_{2}^{13}$ & $\begin{array}{l}P m \\
C_{s}^{1} \\
m P 8\end{array}$ & $\begin{array}{l}a=2 a_{d}[100] \\
b=2 a_{d}[010] \\
c=c_{d}[001]\end{array}$ & $\begin{array}{l}(N / 8)\left[4 W_{A B}^{1}+3 W_{B C}^{1}+\right. \\
3 W_{C A}^{1}+5 W_{A B}^{2}+2 W_{B C}^{2}+ \\
\left.2 W_{C A}^{2}+3 W_{B C}^{3}+3 W_{C A}^{3}\right]\end{array}$ \\
\hline \multicolumn{4}{|c|}{$P_{C}^{1}=P_{C}^{6}=1, P_{B}^{2}=P_{B}^{3}=P_{B}^{7}=1, P_{A}^{4}=P_{A}^{5}=P_{A}^{8}=1$} \\
\hline$A_{3} B_{3} C_{2}^{14}$ & $\begin{array}{l}P m \\
C_{s}^{1} \\
m P 8\end{array}$ & $\begin{array}{l}a=2 a_{d}[100] \\
b=2 a_{d}[010] \\
c=c_{d}[001]\end{array}$ & $\begin{array}{l}(N / 8)\left[4 W_{A B}^{1}+3 W_{B C}^{1}+\right. \\
3 W_{C A}^{1}+3 W_{A B}^{2}+2 W_{B C}^{2}+ \\
\left.2 W_{C A}^{2}+6 W_{A B}^{3}+3 W_{B C}^{3}+3 W_{C A}^{3}\right]\end{array}$ \\
\hline \multicolumn{4}{|c|}{$P_{C}^{1}=P_{C}^{6}=1, P_{B}^{2}=P_{B}^{3}=P_{B}^{8}=1, P_{A}^{4}=P_{A}^{5}=P_{A}^{7}=1$} \\
\hline$A_{3} B_{3} C_{2}^{15}$ & $\begin{array}{l}P m \\
C_{s}^{1} \\
m P 8\end{array}$ & $\begin{array}{l}a=2 a_{d}[100] \\
b=2 a_{d}[010] \\
c=c_{d}[001]\end{array}$ & $\begin{array}{l}(N / 8)\left[4 W_{A B}^{1}+3 W_{B C}^{1}+\right. \\
3 W_{C A}^{1}+4 W_{A B}^{2}+3 W_{B C}^{2}+ \\
\left.W_{C A}^{2}+3 W_{A B}^{3}+6 W_{C A}^{3}\right]\end{array}$ \\
\hline$P_{C}^{1}=P_{C}^{6}=1$ & $P_{B}^{7}=1, P_{A}^{2}=P_{A}^{s}=$ & & \\
\hline
\end{tabular}

As mentioned earlier, it is difficult to determine all the ground-state structures in view of the large number of parameters involved. However, this can be attempted under some assumptions which reduce the number of parameters. Let the axial ratio be close to ideal and the third neighbour interchange energies be negligible. 


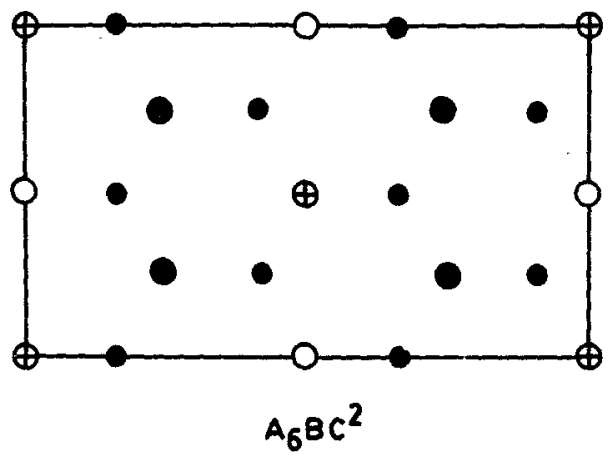

Figure 6. Basal plane projection of $A_{6} B C^{2}$ structure. $A, B$ and $C$ atoms are represented by $O$, $O$ and $\oplus$ respectively.

These assumptions imply that $W_{A B}^{1} \simeq W_{A B}^{2}, W_{B C}^{1} \simeq W_{B C}^{2}, W_{A C}^{1} \simeq W_{A C}^{2}$ and $W_{A B}^{3}$ $\simeq W_{B C}^{3} \simeq W_{A C}^{3} \simeq 0$. Using these values, we have simplified the energy expressions for the different stoichiometric structures (tables 4-8). A consideration of these energy expressions shows that, in several instances, ordered phases with the same stoichiometry but different structures e.g. $A_{6} B C^{2}$ and $A_{6} B C^{3}$, have the same energy. This is the second type of degeneracy noted in the present study and occurs for ideal hcp ternary alloys. Such a degeneracy is lost, when third neighbour interactions are considered. Subsequently, we selected all the energetically different structures for each stoichiometry and focused our attention on the determination of the domains (ranges of $w_{1}=W_{B C}^{1} / W_{A B}^{1}$ and $w_{2}=W_{A C}^{1} / W_{A B}^{1}$ ) of stability of all structures. Structures having lowest energy within their class (stoichiometry) were found and the corresponding domain of $w$ 's identified for negative value of $W_{A B}^{1}$ by drawing iso-energy lines as explained below. For example, under the assumptions mentioned earlier, there are only 6 energetically distinct structures for the stoichiometry $A_{3} B_{3} C_{2}$ in contrast to the 15 given in table 7 . On equating the energies of these structures in pairs, iso-energy lines for each pair were obtained. A plot of these iso-energy lines on a plot with $w_{1}$ and $w_{2}$ as axes leads to different domains in which one of the structures (amongst the six) has lowest energy. As shown in figure 7 only four structures, namely, $A_{3} B_{3} C_{2}^{1}, A_{3} B_{3} C_{2}^{2}, A_{3} B_{3} C_{2}^{3}$ and $A_{3} B_{3} C_{2}^{4}$ have lowest energy in the four different domains. A similar procedure was followed for each stoichiometry and this resulted in two $A_{6} B C\left(A_{6} B C^{1}\right.$ and $\left.A_{3} B C^{2}\right)$, two $A_{5} B_{2} C\left(A_{5} B_{2} C^{1}\right.$ and $\left.A_{5} B_{2} C^{2}\right)$, two $A_{4} B_{3} C\left(A_{4} B_{3} C^{2}\right.$ and $\left.A_{4} B_{3} C^{3}\right)$, three $A_{4} B_{2} C_{2}\left(A_{4} B_{2} C_{2}^{1}, A_{4} B_{2} C_{2}^{2}\right.$ and $\left.A_{4} B_{2} C_{2}^{3}\right)$ and four $A_{3} B_{3} C_{2}\left(A_{3} B_{3} C_{2}^{1}, A_{3} B_{3} C_{2}^{2}\right.$, $A_{3} B_{3} C_{2}^{3}$ and $A_{3} B_{3} C_{2}^{4}$ ) low-energy ordered structures. The assumptions under which these ordered structures have been shown to have low energies will be valid to only a limited extent in real structures. However, lacking more complete specification of all the interchange energies, they provide some insight regarding the possible stable structures.

All the 48 structures mentioned above can also be generated by substituting some atoms with $C$ atoms in binary ground-state structures, obtained by eight sublattice division (Singh and Lele 1990). For example, if we start with $A_{3} B^{1}$, the $D O_{19}$ type of structure, and replace half the $B$ atoms by $C$ atoms, we get $A_{6} B C^{1}$ structure. Similarly, by replacement of some of the $A$ atoms by $C$ atoms in $A_{3} B^{1}$ binary alloy, 


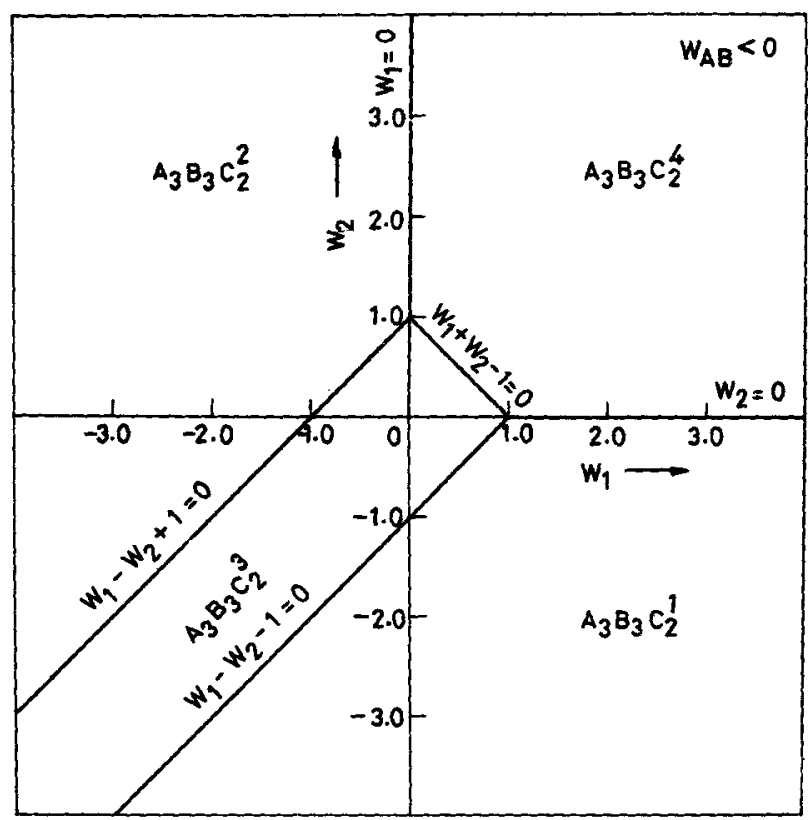

Figure 7. Stability regions of different low energy ordered structures with stoichiometry $A_{3} B_{3} C_{2}$ as a function of interchange energy ratios $w_{1}$ and $w_{2}$.

one can obtain $A_{5} B_{2} C^{2}, A_{4} B_{2} C_{2}^{1}, A_{4} B_{2} C_{2}^{3}$ and $A_{4} B_{2} C_{2}^{15}$ structures. Some structures can also be generated by simultaneous replacement of appropriate fractions of $A$ and $B$ atoms in binary alloys by $C$ atoms. We found a large number of structures with $A B$ stoichiometry in our earlier studies (Singh and Lele 1990) which have greater energy than the $A B^{1}, A B^{2}, A B^{3}, A B^{4}$ and $A B^{5}$ structures. We also note that $A B^{1}, A B^{2}$ and $A B^{3}$ structures are obtained by eight sublattice division considered in the present investigation. The other $A B$ stoichiometric structures obtained by this procedure can also be used to generate ternary structures, for example, $A_{4} B_{3} C^{2}$, $A_{4} B_{3} C^{5}$ and $A_{4} B_{2} C_{2}^{2}$. It is difficult to predict the stability of these structures; however, ternary additions may possibly stabilize these structures.

Banerjee et al (1988) have recently reported $\mathrm{Ti}_{2} \mathrm{AlNb}$ (space group $\mathrm{Cmcm}$ ) phase which precipitates out from $\alpha-\mathrm{Ti}_{3} \mathrm{Al}\left(\mathrm{DO}_{19}\right.$ ) base ternary alloy $\mathrm{Ti}-25$ at. $\% \mathrm{Al}-12$ at. $\% \mathrm{Nb}$. They assigned the space group and also determined the atomic positions using convergent beam electron diffraction and channelling microanalysis. This result has been confirmed by Mozer et al (1990) using neutron powder diffraction. They concluded that ordering causes breaking of the hexagonal symmetry accompanied by distortion of the unit cell. A contraction of $b$ and expansion of $a$ parameters occurs while $c$ parameters of both phases are the same. This structure corresponds to our low energy $A_{4} B_{2} C_{2}^{3}$ structure which, as mentioned earlier, can be derived from the ground state $A_{3} B^{1}$ structure. This former structure has minimum energy for $W_{A B}^{1}<0, w_{1}>0$ and $w_{2}>0$. This means that all first neighbour interchange energies are attractive and stabilize the $A_{4} B_{2} C_{2}^{3}$ structure. It is clear that $C(\mathrm{Nb})$ atoms replace some $A(\mathrm{Ti})$ atoms preferentially to stabilize this structure. Hence, if we know the initial binary phase, it is possible to predict the different ternary structures formed by suitable ternary additions. 


\section{Conclusions}

(i) Nine energetically distinct ground-state ordered structures with the stoichiometries $A_{12} B C, A_{7} B_{6} C, A_{10} B C, A_{6} B_{5} C, A_{5} B_{4} C, A_{6} B C, A_{4} B_{3} C, A_{7} B_{5} C_{2}$ and $A_{3} B_{2} C$ have been found.

(ii) Fortyeight ordered structures have been identified in ternary hep alloys on the basis of a division of the disordered structure into 8 sublattices.

(iii) The structures have Bravais lattices which are hexagonal, orthorhombic or monoclinic.

(iv) Thirteen ordered structures (of 48) have low energies under the assumptions that the axial ratio is close to ideal and third neighbour interchange energies are negligible.

(v) Two types of degeneracy have been found. One type of degeneracy occurs for ideal hcp alloys where only first neighbour interactions are considered. Another degenerate situation occurs with the ground state structures even when third neighbour interactions are considered.

\section{Acknowledgements}

The authors wish to acknowledge the financial support from the Department of Science and Technology, New Delhi through a project. They also acknowledge the helpful discussions with Professor Vakil Singh.

\section{References}

Allen S M and Cahn J W 1972 Acta Metall. 20423

Banerjee D, Gogia A K, Nandy T K and Joshi V A 1988 Acta Metall. 36871

Hahn T 1989 International tables for crystallography, Volume A (The Netherlands: Kluwer Academic Publishers)

Kanamori J 1966 Prog. Theor. Phys. 3516

Kudō T and Katsura S 1976 Prog. Theor. Phys. 56435

Mozer B, Bendersky L A, Boettinger W J and Rowe R G 1990 Scr. Met. 242363

Richards M J and Cahn J W 1971 Acta Metall. 191263

Singh A K and Lele S 1990 Philos. Mag. B (Accepted) 